\title{
Transport characteristics of nanoparticle-based ferrofluids in a gel model of the brain
}

\section{Soubir Basak' \\ David Brogan² \\ Hans Dietrich ${ }^{2}$ \\ Rogers Ritter ${ }^{3}$ \\ Ralph G Dacey² \\ Pratim Biswas'}

'Aerosol and Air Quality Research Laboratory, Department of Energy, Environmental and Chemical

Engineering, Washington University in St. Louis, St. Louis, MO, USA;

${ }^{2}$ Department of Neurological Surgery,

Washington University School

of Medicine, St. Louis, MO, USA;

${ }^{3}$ Stereotaxis Inc., St. Louis, MO, USA

Correspondence: Pratim Biswas

Aerosol and Air Quality Research

Laboratory, Department of Energy,

Environmental and Chemical Engineering,

Washington University in St. Louis,

St. Louis, MO 63। 30, USA

$\mathrm{Tel}+|3| 49355548$

Fax +I 3149355464

Email pratim.biswas@wustl.edu

\begin{abstract}
A current advance in nanotechnology is the selective targeting of therapeutics by external magnetic field-guided delivery. This is an important area of research in medicine. The use of magnetic forces results in the formation of agglomerated structures in the field region. The transport characteristics of these agglomerated structures are explored. A nonintrusive method based on in situ light-scattering techniques is used to characterize the velocity of such particles in a magnetic field gradient. A transport model for the chain-like agglomerates is developed based on these experimental observations. The transport characteristics of magnetic nanoparticle drug carriers are then explored in gel-based simulated models of the brain. Results of such measurements demonstrate decreased diffusion of magnetic nanoparticles when placed in a high magnetic field gradient.
\end{abstract}

Keywords: nanoparticle ferrofluid, gel-brain model, drug delivery, magnetic agglomeration, transport, magnetic fields

\section{Introduction}

The ability to precisely target and control the release of a drug to any tissue or region in the body with great specificity is an important objective of therapeutic delivery. Systematic research over the past few decades has been performed to use magnetic fields to steer and target drugs using ferrofluids that can reversibly bind therapeutic agents. ${ }^{1,2}$ The concept of magnetically guided targeted drug delivery is simple, wherein the drug of choice is bound to a particle with a magnetic core, and an external magnet is used to guide the composite to the desired point in the body and/or deposit in a certain location for controlled release of the drug. Many studies have been done to examine the targeting of therapeutics in animal models. ${ }^{3,4}$ Lubbe and colleagues ${ }^{5}$ were the first group to perform a clinical trial using magnetic drug targeting in a small population of 14 patients with advanced solid tumors. Hafeli ${ }^{6}$ has highlighted the requirement of a large and precisely defined magnetic field gradient, as well as the need for the use of small sized particles with a high magnetic susceptibility. Though larger sized particles experience a higher magnetic force (magnetic moment is proportional to the third power of diameter), they are also cleared more rapidly from the blood by the body's reticulo-endothelial system (RES). ${ }^{7}$ Mykhaylyk and colleagues ${ }^{8}$ suggested that particles should be smaller than $400-500 \mathrm{~nm}$, and perhaps even as small as $150 \mathrm{~nm}$ to avoid fenestrations in the liver and spleen. Thus, finding an optimal size is essential and ideal carriers should be as large as possible (to experience larger magnetic forces) but small enough to move into the desired target region and avoid clearance.

The capacity to direct therapeutic agents to specific areas in the brain is important in the treatment of a number of diseases such as Parkinson's disease and cancer.' Much of the current research in this area centers on convection-enhanced delivery (CED), which utilizes positive pressure infusion directly into the target site, but lacks specificity of the volume of distribution. ${ }^{10,11}$ The combination of CED and use of 
magnetic forces to enhance targeting may enable delivery of particles to specific regions or help restrict the particles within pre-defined boundaries, thus, effectively reducing clearance and minimizing adverse interactions with healthy tissue. ${ }^{12-14}$ To accomplish this, the magnetic force generated on the particles would need to be sufficient to overcome diffusion and convective flow in the brain. The use of chaining and the creation of particle agglomerates for therapeutic delivery has been reported by Forbes. ${ }^{12}$ They used superparamagnetic particles that agglomerated in the magnetic field gradient, and thus overcame convective and diffusive forces to remain in the blood vessel and eventually deposit on the walls.

To effectively develop such a system, it is important to evaluate the transport characteristics by understanding the various forces acting on the particles. The velocity of the nanoparticles are dependent on the magnetic and drag forces that act on the particles. ${ }^{13}$ An additional complexity that needs to be accounted for is that magnetic particles tend to agglomerate in a magnetic field gradient and create larger chain-like structures. ${ }^{14-17}$ If the force balance calculations are done based on single particle analysis, ${ }^{18,19}$ the effective magnetic force and velocity of the particles will be underpredicted. While there have been experimental demonstrations of such strategies, ${ }^{20}$ no detailed studies have been conducted to develop an understanding of the increased magnetic forces due to agglomeration and the reduction of transport.

This paper reports a detailed evaluation of velocity of magnetic particles undergoing simultaneous agglomeration in a magnetic field gradient. The change in morphology (shape) of the resultant agglomerate is accounted for in the evaluation. The first experiment evaluated the velocity by monitoring the particle agglomeration state using in situ light-scattering measurements. The second set of experiments demonstrated the formation of such chain-like structures in a gel model of the brain, and demonstrated the potential for targeted delivery by overcoming convective and diffusive forces. A theoretical model is developed to estimate the agglomeration of magnetic nanoparticles and calculate the effective force acting on the nanoparticles due to the change in effective size. The model is used to explain the potential of developing a process for locking magnetic nanoparticles in a certain region after infusion into the brain.

\section{Materials and methods}

\section{Ferrofluids used}

A ferrofluid is a liquid composed of nanoscale ferromagnetic or ferrimagnetic particles suspended in a carrier fluid, usually an organic solvent or water. Often these particles are coated with organic polymers like polyethylene glycol (PEG) to prevent agglomeration and improve surface functionality for potential multimodal biomedical applications. Two types of nanoparticles with different sizes, morphologies and magnetic properties suspended in water were used in this study to create the ferrofluid. The first nanomaterial was clustered $\mathrm{Fe}_{3} \mathrm{O}_{4}$ encapsulated in a biodegradable polymer matrix (PEG-300, MicroMod Partikeltechnologie GmbH, Germany). Two different sizes, $130 \mathrm{~nm}$ (Product No. 09-54-132) and $250 \mathrm{~nm}$ (Product No. 09-54-252) were used. The second nanomaterial was $110 \mathrm{~nm}$ pure uncoated cupro-spinel $\left(\mathrm{CuFe}_{2} \mathrm{O}_{4}\right)$ particles synthesized by spray pyrolysis in a furnace aerosol reactor starting with a 1:2 molar ratio of premixed $\mathrm{CuSO}_{4}$ and $\mathrm{Fe}\left(\mathrm{NO}_{3}\right)_{3}$ in aqueous solution. Details of the synthesis method are provided in the supporting document section. The concentration of particles was diluted using double deionized water to $1 \mathrm{mg} \mathrm{ml}^{-1}$ calculated based on total solid mass of the nanoparticles for all the experiments unless otherwise mentioned. Other characteristics of the particles used are listed in Table 1.

\section{Experimental setup to estimate the velocity of nanoparticles in a magnetic field gradient by an in situ light-scattering technique}

A permanent magnet $(7.62 \times 10.16 \times 12.70 \mathrm{~cm})$ was utilized to create the magnetic field gradient by using the equipolar xy-plane on a nonpole face of the magnet. The change in magnetic flux density and magnetic field gradient as a function of

Table I Size and magnetic properties of nanomaterials used

\begin{tabular}{|c|c|c|c|c|c|c|}
\hline Material & $\begin{array}{l}\text { Mean primary } \\
\text { particle size }(\mathrm{nm})\end{array}$ & Composition & $\begin{array}{l}\text { Density } \\
\left(\mathrm{kg} \mathrm{m}^{-3}\right)\end{array}$ & $\begin{array}{l}\text { Moment } \\
\left(\text { emu gm }{ }^{-1} @ \text { I kOe) }\right.\end{array}$ & $\begin{array}{l}\text { Saturated moment } \\
\left(\text { emu gm }{ }^{-1} @ 10 \text { kOe) }\right.\end{array}$ & $\begin{array}{l}\chi_{v}(\text { Volumetric magnetic } \\
\text { susceptibility @ I kOe) }\end{array}$ \\
\hline 1 & 250 & $\begin{array}{l}\text { PEG-coated } \\
\mathrm{Fe}_{3} \mathrm{O}_{4}\end{array}$ & ${ }^{\mathrm{a}} 2500$ & ${ }^{\mathrm{a}} 43.0$ & ${ }^{\mathrm{a}} 67.0$ & 1.351 \\
\hline 2 & 130 & $\begin{array}{l}\text { PEG-coated } \\
\mathrm{Fe}_{3} \mathrm{O}_{4}\end{array}$ & a 3000 & ${ }^{\mathrm{a}} 43.0$ & ${ }^{\mathrm{a}} 67.0$ & 1.621 \\
\hline 3 & 110 & $\mathrm{CuFe}_{2} \mathrm{O}_{4}$ & b5400 & c27.5 & c38.6 & 1.866 \\
\hline
\end{tabular}

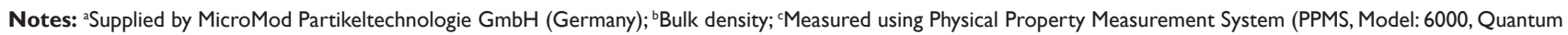
Design). 
distance from its surface was measured by a Lakeshore 421 Gauss meter with MMA-2502-VH Hall probe (Figure S2).

The velocity of the particles in the magnetic field gradient were measured in an aqueous suspension using an in situ static light-scattering technique similar to that described by Kulkarni and colleagues. ${ }^{20}$ The optical system is comprised of a tungsten-filament lamp, a $455 \mathrm{~nm}$ filter, lenses and apertures to focus the light, along with a detector placed at $90^{\circ}$ with respect to incident beam to monitor scattered light (Figure 1). $20 \mathrm{ml}$ ferrofluid $\left(1 \mathrm{mg} \mathrm{ml}^{-1}\right)$ was placed in a Borosilicate glass sample cell $(9.5 \mathrm{~cm}$ high $\times 2.5 \mathrm{~cm}$ inner diameter) with rubber-lined screw caps. The magnet was placed below the sample cell at different distances to vary the magnetic field gradient. The intensity of the scattered light was measured at 10 s time intervals after imposition of the magnetic field gradient. The scattering intensity was measured at three different field gradients $(4.1,3.6$, and 2.0 $\mathrm{T} \mathrm{m}^{-1}$ ) and this varied due to collision and agglomeration of particles. Three to five measurements were taken for each ferrofluid at each magnetic field gradient for statistically relevant results. The travel path length for the ferrofluid material was kept constant $(1.1 \mathrm{~cm})$ and the mean time to traverse the path was estimated from the time taken to lose $50 \%$ of the scattering intensity (midpoint of the slope of the normalized scattering intensity versus time plot). The average velocity $\left(v_{e}\right)$ of the particles in the ferrofluid was calculated by dividing the path length traversed by the mean time taken. Based on the experimentally determined velocity of the ferrofluid particles in the magnetic field gradient, a theory is proposed to estimate the average number of particles in single agglomerate, and to calculate the effective magnetic force exerted on the nanoparticle agglomerates.

To support the formation of a chain-like agglomerated structure in the presence of a magnetic field, scanning electron microscope (SEM, model Hitachi S4500) images were taken of the particles. The SEM samples were prepared by taking $0.5 \mathrm{ml}$ of ferrofluid on a SEM mount (Ted Pella Inc., product ID 16202). The SEM mount was then placed in a magnetic field gradient and kept for $24 \mathrm{hr}$ to evaporate the solvents. The dry nanoparticles on the SEM mount were imaged to analyze the morphology.

\section{Experimental setup to establish the transport of particles in a gel model using light microscopy}

The understanding of particle transport characteristics gained in the preceding experiments was applied to study particle transport behavior in a gel model of the human brain. A $0.6 \%$ agarose gel was selected as the in vitro model for the experiment, and has been previously validated by infusion experiments within a porcine brain. ${ }^{21}$ In those experiments, the gel was found to have a distribution to infusion volume ratio $28 \%$ greater than the porcine brain; with the conclusion made that the gel's hydraulic conductivity was roughly $1 / 3$ greater than that of the brain. ${ }^{21}$ Measurements of inter-fiber distance from electron micrographs ${ }^{22}$ show a distance of $480 \mathrm{~nm}$ for a $0.6 \%$ agarose gel.

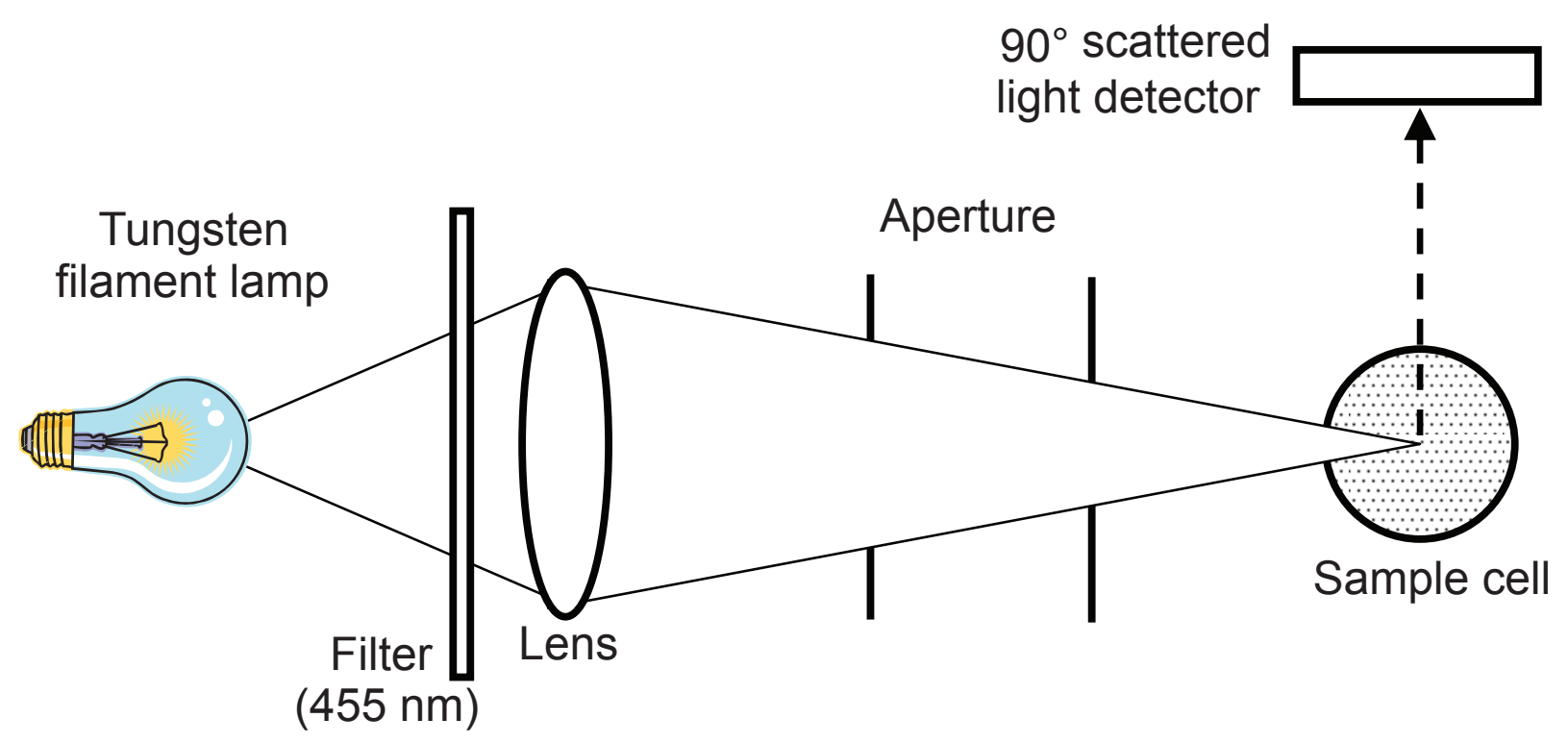

Figure I Experimental setup to study the transport characteristic of the ferrofluid in a controlled environment. The magnet was placed below the sample cell at different distances to vary the magnetic field gradient. 
Incidental observation of ferrofluid in the presence of the magnetic field gradient suggested that strong magnetic fields might hinder transport of nanoparticles within the gel due to particle agglomeration. Therefore, an experiment was devised to assess the effects of the magnetic field gradient on transport of magnetic nanoparticles inside the pores of a gel. The gel was made by mixing agarose powder (Sigma Aldrich, St. Louis, MO) with $1 \mathrm{X}$ TBE as described by Chen and colleagues ${ }^{21}$ and heating in the microwave until fully dissolved. The gel was removed, allowed to cool for five minutes and placed into $15 \mathrm{ml}$ plastic test tubes.

A microinjection pump and polyethylene tubing (Intramedic) with $1.22 \mathrm{~mm}$ outside diameter was used to infuse $1 \mathrm{mg} \mathrm{ml}^{-1}$ of $250 \mathrm{~nm}$ particles (material 1) suspended in water into the test tubes filled with $3 \mathrm{ml}$ of gel. A total of $10 \mu \mathrm{l}$ of infusate was delivered at a rate of $2 \mu \mathrm{min}^{-1}$ into the gel. $1 \mathrm{ml}$ of deionized water was placed onto the top of the gel to prevent dehydration and provide a small hydrostatic pressure to enhance transport. Four gels were placed onto the magnet with the bottom of the test tube resting above the pole of the magnet, with the vertical axis in the direction of the field lines. For the high field experiment, the particles were exposed to a gradient of $8.0 \mathrm{~T} \mathrm{~m}^{-1}$ while in the low field experiment the particles experienced a gradient of $0.55 \mathrm{~T} \mathrm{~m}^{-1}$. The control group consisted of four gels placed outside of the magnetic field gradient in the same vertical orientation as the experimental gels.

An image of each gel was taken after infusion of the ferrofluid and the orientation of the test tube, along with its position in the field, was recorded to ensure reproducibility. Images were obtained via a Zeiss light microscope equipped with a Dage-MTI CCD-72 camera. At the lowest power objective, a resolution of approximately $5 \mu \mathrm{m}$ pixel $^{-1}$ was achieved. An image from each gel and time point was inverted so that the dark particles corresponded to higher pixel values than the background. The mean and median pixel value of each image was then calculated for a $625 \times 415$ pixel area. The mean and median pixel value was then normalized for each gel, with the $0 \mathrm{hr}$ value set as the baseline. All images were analyzed with NIH ImageJ Version 1.36b. It is important to note that while the gels resided for most of the experiment in the vertical plane, the microscope setup required that they be turned horizontally to be imaged. Gels were removed from the magnet and control area for imaging at 3,6, and 16 hours and then every 16 hours after that until the 48 hour mark. The experiment was repeated with the $130 \mathrm{~nm}$ particles (material 2) placed in both the high field and the low field regions along with the control group.

\section{Results and discussion}

A theory is proposed to estimate the particle agglomeration and effective force taking into account the agglomerated chain-like structure. The velocity of nanoparticles in an external magnetic field gradient was determined by a static light-scattering technique. The experimentally measured velocity was used to estimate the effective particle size due to chaining based on the proposed theory. The transport of nanoparticles in a gel model was then established.

\section{Theoretical estimation of particle agglomeration and effective force acting on particles in ferrofluid in magnetic field gradient}

Two forces act on a single nanoparticle suspended in a fluid medium in the magnetic field gradient - the magnetic force and the drag force. The terminal velocity $\left(v_{t, 1}\right)$ in a magnetic field gradient can be readily obtained based on a single particle force balance as reported by Holligan and colleagues ${ }^{18}$ and Kuhn and colleagues ${ }^{19}$

$$
v_{t, 1}=\frac{\chi_{v} \delta B^{2} d_{p}^{2}}{36 \eta \mu_{0}}
$$

where, $\chi_{v}$ is the volumetric magnetic susceptibility of the nanoparticle, $\delta B$ is the magnetic field gradient, $d_{p}$ is the mean diameter of the primary nanoparticle, $\eta$ is the viscosity of the fluid medium into which the particles are injected, and $\mu_{0}$ is the permeability of free space.

However, when placed in a magnetic field gradient, the ferromagnetic particles in the ferrofluid interact with each other and form agglomerated structures. The morphology of these agglomerates formed by collision of nanoparticles in an external magnetic field gradient typically results in a chain-like structure. Thus the terminal velocity calculated based on the force balance on a single particle is expected to under predict the actual velocity of the particles due to a change in the effective size (that will alter both the magnetic force and the drag force). To account for the chaining of nanoparticles due to collisions in the magnetic field gradient, a modified magnetic force and drag force expression is proposed. Assuming each chain-like agglomerate is made up of an average of " $n$ " number of primary particles with mean diameter $d_{p}$, the modified magnetic force $\left(F_{m, c}\right)$ can be given by,

$$
F_{m, c}=\frac{n \pi \chi_{v} \delta B^{2} d_{p}^{3}}{12 \mu_{0}}
$$


The drag force on a nonspherical structure can be estimated by extending the Navier-Stokes equation for drag on an isolated sphere in creeping flow. ${ }^{23-25}$ A detailed drag force expression proposed by Leith ${ }^{25}$ for nonspherical shape particles is used to estimate the drag force on the chain-like magnetic nanoparticle agglomerate $\left(F_{d, c}\right)$,

$$
F_{d, c}=3 \pi \eta v_{t, c} d_{n}\left(\frac{1}{3}+\frac{2}{3} \frac{d_{n}}{d_{s}}\right)
$$

where $v_{t, c}$ is the terminal velocity for a chain-like structure, $d_{n}$ is the diameter of the sphere with same projected area as the area of the particle projected normal to its direction of motion, $d_{s}$ is the diameter of the sphere whose effective surface equals that of the particle. For a chain-like structure formed by agglomeration of " $n$ " particles in a linear manner with mean diameter of each primary particle being $d_{p}, d_{n}$ can be replaced with $d_{p}$ and $d_{s}$ can be replaced by $n^{1 / 2} d_{p}$. Thus the drag force for a chain-like structure (Eq. 3) becomes

$$
F_{d, c}=3 \pi \eta v_{t, c} d_{p}\left(\frac{1}{3}+\frac{2}{3} \sqrt{n}\right)
$$

The terminal velocity for a chain-like structure $\left(v_{t, c}\right)$ in a magnetic field gradient can be calculated by equating the modified magnetic force (Eq. 2) to drag force (Eq. 4) as

$$
v_{t, c}=\frac{\chi_{v} \delta B^{2} d_{p}^{2}}{36 \eta \mu_{0}}\left(\frac{3 n}{1+2 \sqrt{n}}\right)=v_{t, 1}\left(\frac{3 n}{1+2 \sqrt{n}}\right)
$$

Based on proposed derivation, it is clear, that the terminal velocity for a chain-like agglomerated structure is enhanced by a factor dependent on the number of primary particles in the agglomerate. The theoretically calculated terminal velocity in the magnetic field gradient based on single particle force balance always underestimate the actual velocity of particles in the presence of an external magnetic field gradient. Furthermore, the expression for the terminal velocity for a chain-like structure (Eq. 5) can be solved to estimate the average number of primary particles in agglomerate as
The equation for the number of primary particles in an agglomerate can be further simplified as

$$
n=\frac{1}{9}\left[\xi(3+2 \xi)+2 \sqrt{\xi^{3}(3+\xi)}\right]
$$

where $\xi$ is a dimensionless magnetic velocity enhancement factor given as $v_{t, c} / v_{t, l}$. The estimated number of primary particles in an agglomerate (Eq. 6 or 7) can be used to calculate the effective force acting on a chain-like agglomerate using Equation 2.

The proposed theory based on creeping flow of a nonspherical shape particle falls within the laminar flow region of Reynolds number calculated based on experimental velocity and mean agglomerate particle size. The use of average $n$ number of primary particles in a chain-like structure to calculate the effective magnetic force can be justified by comparing the characteristic time to form the chain-like morphology with that required to attain the terminal velocity in a magnetic field gradient. The characteristic time for terminal velocity in a magnetic field gradient $\left(\tau_{m}\right)$ is calculated by estimating the time to travel a distance of $1 \mathrm{~cm}$, based on a single particle force balance. The characteristic time for forming chain-like structures due to collisions in a magnetic field gradient can be estimated by an analytical expression given by Kumar and colleagues. ${ }^{26}$ Assuming the interacting particles form a chain-like agglomerate in a magnetic field gradient, the collision frequency function in the free molecular regime $\left(\beta_{F M}\right)$ is given by

$$
\begin{gathered}
\beta_{F M}=\frac{2}{3^{4 / 3}}\left[\frac{\pi \chi_{i} \chi_{j} B^{2}}{\mu_{o}}\right]^{1 / 3}\left(k_{B} T\right)^{1 / 6} \frac{1}{p_{p}{ }^{1 / 2}}\left(d_{i} d_{j}\right) \\
{\left[\frac{1}{d_{i}^{3}}+\frac{1}{d_{j}^{3}}\right]^{1 / 2}\left(d_{i}+d_{j}\right)}
\end{gathered}
$$

where $B$ is the magnetic flux density, $k_{B}$ is the Boltzmann constant, $T$ is the temperature, and $\rho_{p}$ is the density of the nanoparticles. The expression for the collision frequency function (Eq. 8) can be further simplified based on a monodisperse particle assumption (ie, $d_{i}=d_{j}=d_{p}$ and $\left.\chi_{i}=\chi_{j}=\chi_{v}\right)$

$$
\beta_{F M}=\frac{4}{3^{4 / 3}}\left[\frac{\pi \chi_{v} B^{2}}{\mu_{0}}\right]^{1 / 3}\left(2 k_{B} T\right)^{1 / 6} \frac{1}{\rho_{p}{ }^{1 / 2}} d_{p}^{3 / 2}
$$

$$
n=\frac{12\left[v_{t, c} \eta \mu_{0}\left(24 v_{t, c} \eta \mu_{0}+\delta B^{2} d_{p}{ }^{2} \chi_{v}\right)+4 \sqrt{3} \sqrt{v_{t, c}{ }^{3} \eta^{3} \mu_{0}{ }^{3}\left(12 v_{t, c} \eta \mu_{0}+\delta B^{2} d_{p}{ }^{2} \chi_{v}\right)}\right]}{\delta B^{4} d_{p}{ }^{4} \chi_{v}{ }^{2}}
$$


The characteristic time for magnetic coagulation $\left(\tau_{c}\right)$ can then be calculated as $\tau_{c}=1 /\left(N_{o} \beta_{F M}\right)$, where $N_{\mathrm{o}}$ is the initial concentration of nanoparticles.

\section{Experimental measurement of velocity of particles in a ferrofluid in a magnetic field gradient by in situ static light scattering technique: Estimation of chaining and effective magnetic force}

The intensity of scattered light for the $110 \mathrm{~nm}$ ferrofluid was normalized to initial time $(\mathrm{t}=0 \mathrm{~s})$ and plotted against time (Figure 2). There are two clearly distinguishable plateaus that can be identified in this figure. The decrease in normalized intensity (normalized at $\mathrm{t}=0 \mathrm{~s}$ ) followed by the plateau at $\mathrm{t}<100 \mathrm{~s}$ is thought to be caused by changes in the morphology of the agglomerated particles due to the external magnetic field gradient (Figure S3). When initially placed in a magnetic field gradient, the particles tend to form an ordered chain-like morphology compared to its initial random unagglomerated morphology. A drop in scattering intensity is expected due to this morphology change that results in a decrease in the particle number concentration. The continued decrease in normalized intensity (normalized at $t=100 \mathrm{~s}$ ) followed by the second plateau at $t>100 \mathrm{~s}$ results from a decrease in the absolute concentration of the particles due to magnetic mobi- lization and settling of the ferrofluid particles (Figure S4). The drop in scattering intensity due to formation of chain-like structures due to collision of particles in the magnetic field gradient is a reversible process (on removal of field), while the drop in scattering intensity due to settling is irreversible (loss of particles from view volume). The time scale $\left(\tau_{c}\right)$ to reorganize the morphology by collision of particles for all materials in different external magnetic fields were theoretically calculated and plotted in Figure 3 based on the properties of the materials listed in Table 1. The calculated characteristic time for agglomeration due to collisions was found to be on the order of $\sim 10^{-3} \mathrm{~s}$, which is similar to the experimentally measured value by Fernandes and colleagues. ${ }^{27}$ The estimated characteristic time for velocity $\left(\tau_{m}\right)$ in an external magnetic field gradient is on the order of $\sim 10^{3} \mathrm{~s}$ (Figure 3 ). The order of magnitude analysis of the characteristic time for agglomeration by collisions and particle velocity clearly justifies the use of a chain-like structure to estimate the true velocity of chain-like agglomerates. It also suggests that the two plateaus found in the plot of normalized scattering intensity versus time (Figure 2) are caused by two different phenomena: the first one due to collisional growth in magnetic fields, while the second one is due to settling under the influence of the magnetic field gradient. The same trend is seen in the plot of initial normalized scattering intensity versus time for the other two materials (Figures S5 and S6).

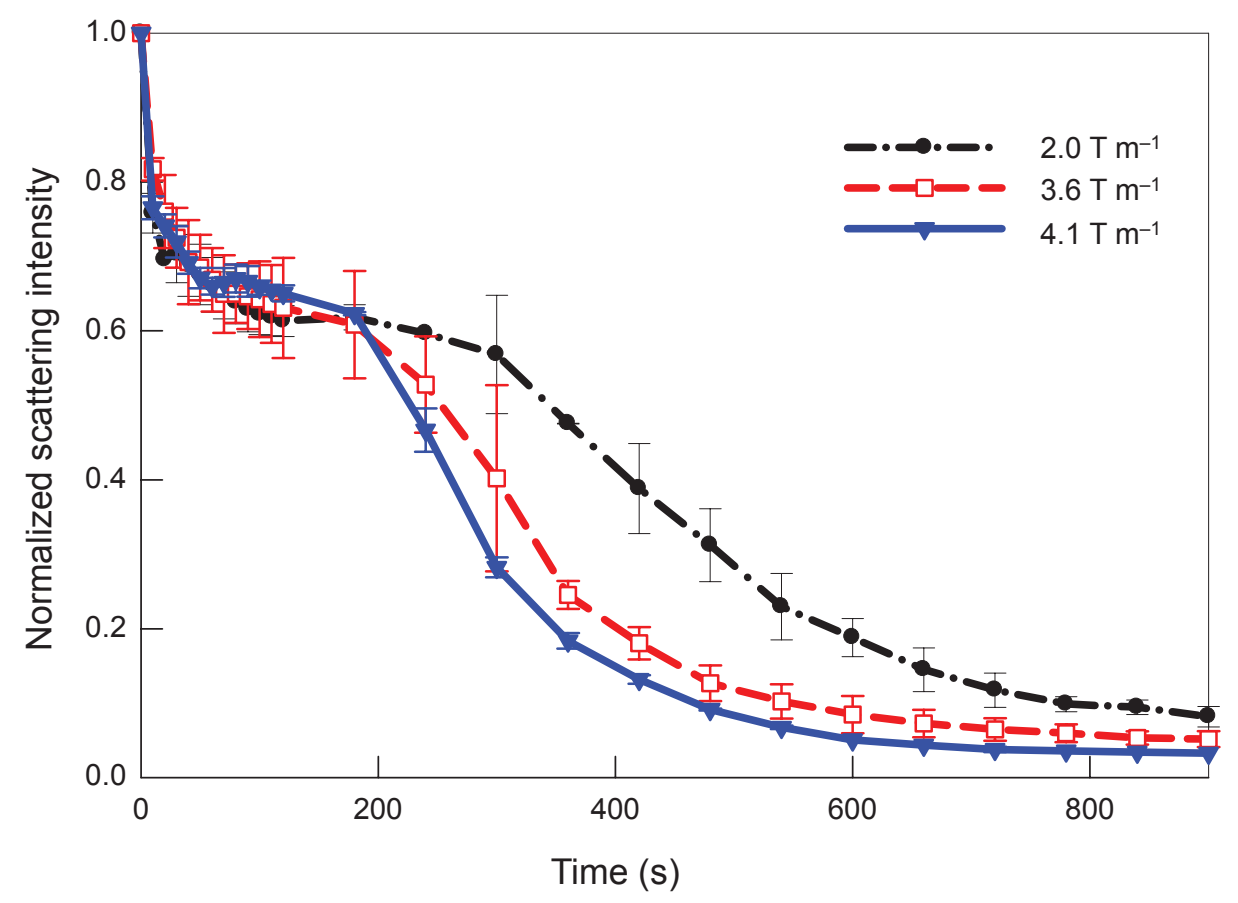

Figure 2 Variation of normalized scattering intensity (normalized at $\mathrm{t}=0 \mathrm{~s}$ ) with time for material $3(1 \mathrm{I} 0 \mathrm{~nm}$ ferrofluid) at various applied magnetic field gradient. 


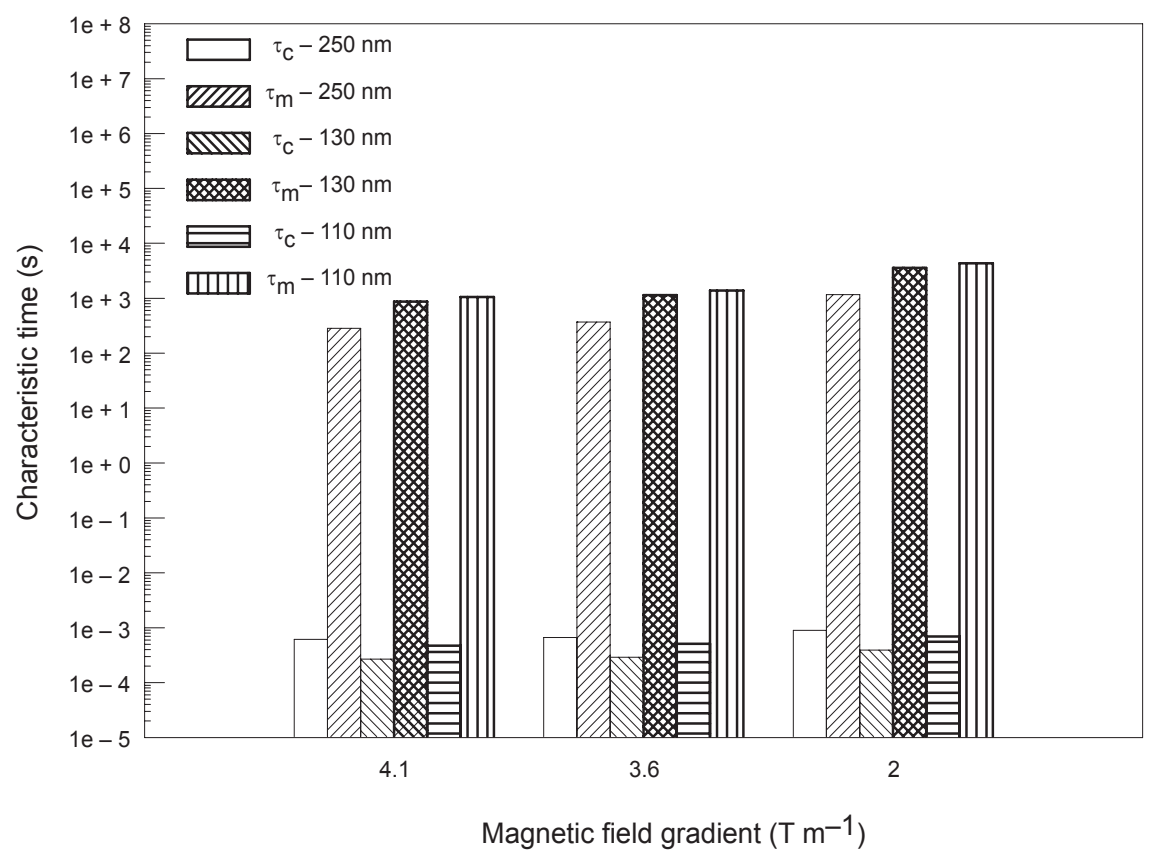

Figure 3 Comparison of characteristic time of collisional growth in magnetic field $\left(\tau_{c}\right)$ and characteristic time to traverse $\mathrm{I} \mathrm{cm}\left(\tau_{\mathrm{m}}\right)$ for different ferrofluids at various magnetic field gradients.

Variation of scattering intensity normalized to $\mathrm{t}=100 \mathrm{~s}$ (Figure S3) is used to estimate the velocity of $110 \mathrm{~nm}$ particles in the external magnetic field gradient listed in Table 2. Similarly, the velocity of $130 \mathrm{~nm}$ and $250 \mathrm{~nm}$ particles in the ferrofluid is estimated from the plot of scattering intensity normalized to $\mathrm{t}=30 \mathrm{~s}$ (Figure 4 ). The scattering intensity normalized to $\mathrm{t}=0 \mathrm{~s}$ for these materials are provided in Supporting information (Figures S5 and S6). A different time is used to normalize the scattering intensity for the $130 \mathrm{~nm}$ and $250 \mathrm{~nm}$ ferrofluid $(\mathrm{t}=30 \mathrm{~s})$ compared to $110 \mathrm{~nm}$ ferrofluid $(\mathrm{t}=100 \mathrm{~s})$. The time is chosen based on experimental measurements close to the actual position of the first plateau. The properties of the materials listed in Table 1 are used in equation 1 to theoretically predict the terminal velocity based on a single particle force balance in water in the presence of an external magnetic field gradient (Table 2). A plot of the experimentally measured and theoretically predicted velocity as a function of the magnetic field gradient is shown in Figure 5. It is of note that the experimentally measured velocity exceeded the predicted values by as little as a factor of $\sim 3$ and as much as a factor of $\sim 10$. This is attributed to the agglomeration of particles while in the magnetic field gradient, giving the particles a larger effective size and therefore experiencing a larger effective

Table 2 Experimental matrix to study the transport characteristic of different ferrofluid in controlled environment

\begin{tabular}{|c|c|c|c|c|c|}
\hline \multirow[t]{2}{*}{ Material } & \multirow{2}{*}{$\begin{array}{l}\text { Magnetic field gradient } \\
\left(T \mathrm{~m}^{-1}\right)\end{array}$} & \multicolumn{2}{|c|}{ Particle velocity $\left(\mathrm{cm} \mathrm{s}^{-1}\right)$} & \multirow[t]{2}{*}{$v_{e} / v_{t}$} & \multirow{2}{*}{$\begin{array}{l}\text { Estimated number of } \\
\text { primary particles in } \\
\text { each agglomerate }(n)\end{array}$} \\
\hline & & Experimental $\left(v_{e}\right)$ & Theoretical $\left(v_{t, 1}\right)$ & & \\
\hline \multirow{3}{*}{ I } & 4.1 & $1.0 \times 10^{-2}$ & $3.5 \times 10^{-3}$ & 3.0 & 6 \\
\hline & 3.6 & $8.5 \times 10^{-3}$ & $2.7 \times 10^{-3}$ & 3.1 & 6 \\
\hline & 2.0 & $5.2 \times 10^{-3}$ & $8.6 \times 10^{-4}$ & 6.1 & 22 \\
\hline \multirow{3}{*}{2} & 4.1 & $4.6 \times 10^{-3}$ & $1.1 \times 10^{-3}$ & 4.0 & 10 \\
\hline & 3.6 & $3.3 \times 10^{-3}$ & $8.8 \times 10^{-4}$ & 3.8 & 9 \\
\hline & 2.0 & $2.0 \times 10^{-3}$ & $2.8 \times 10^{-4}$ & 7.3 & 32 \\
\hline \multirow{3}{*}{3} & 4.1 & $4.6 \times 10^{-3}$ & $9.5 \times 10^{-4}$ & 4.8 & 14 \\
\hline & 3.6 & $3.3 \times 10^{-3}$ & $7.2 \times 10^{-4}$ & 4.6 & 13 \\
\hline & 2.0 & $2.3 \times 10^{-3}$ & $2.3 \times 10^{-4}$ & 10.0 & 58 \\
\hline
\end{tabular}




\section{A}

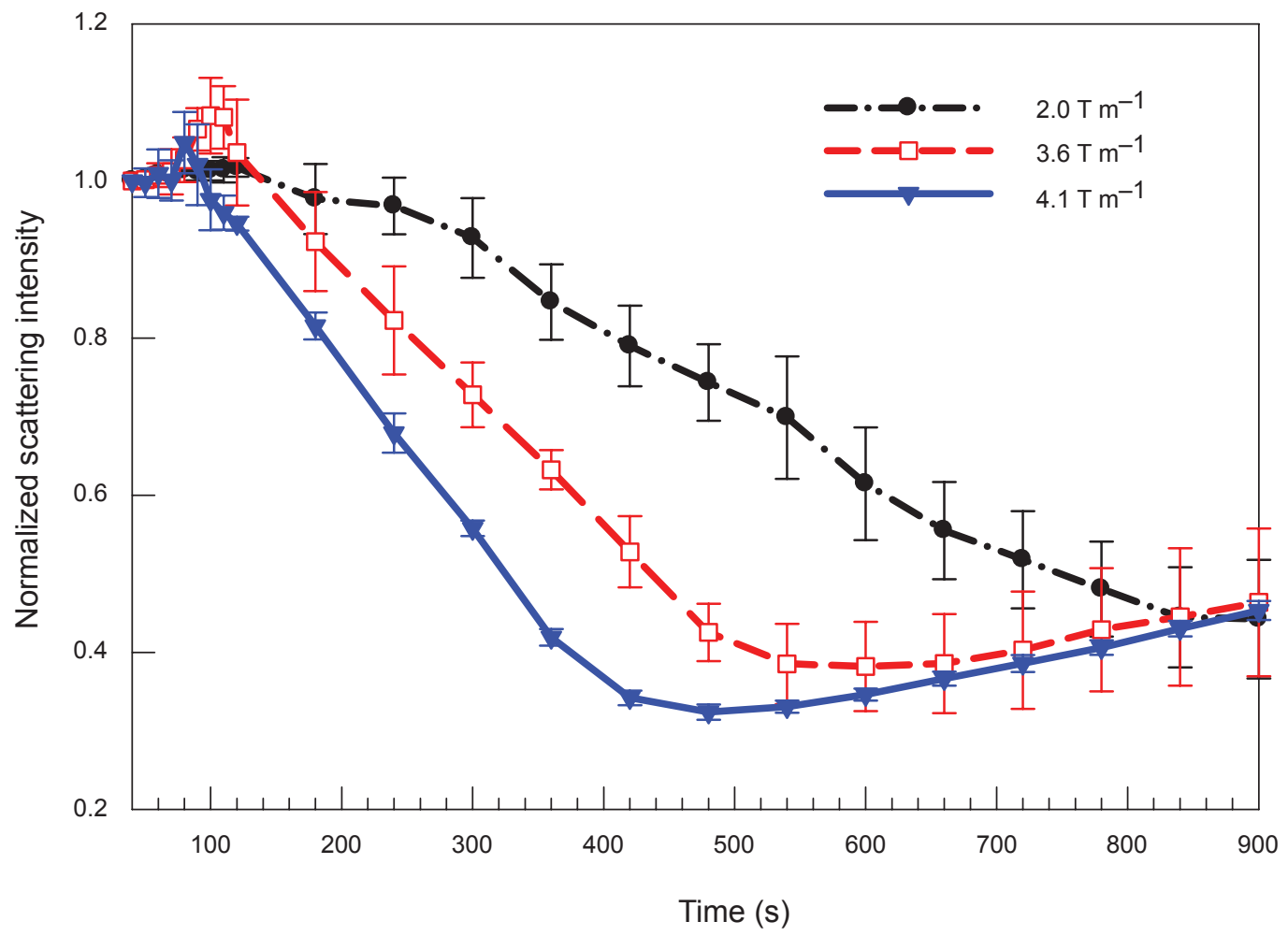

B

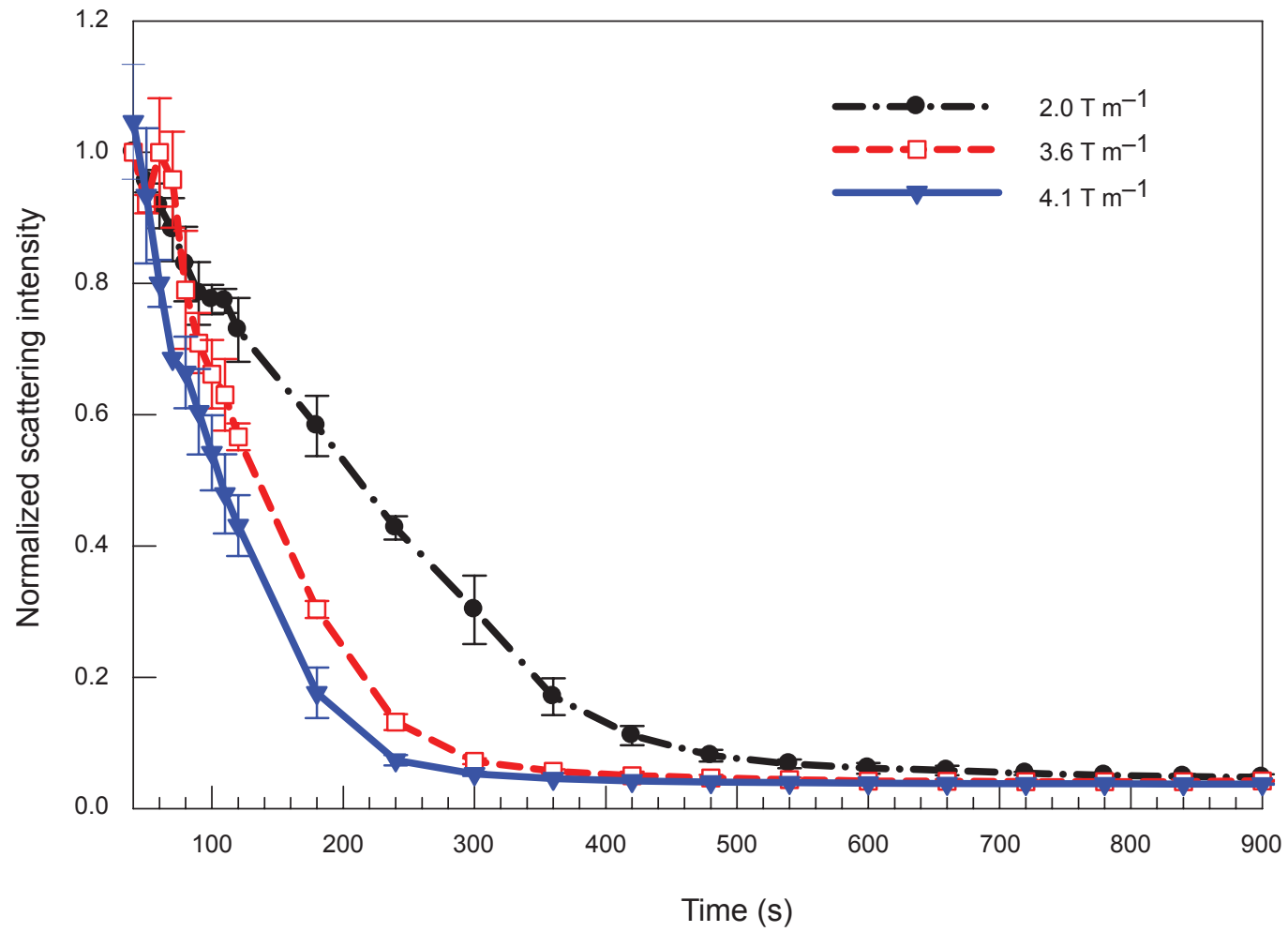

Figure 4 A) Variation of normalized scattering intensity (normalized at $\mathrm{t}=30 \mathrm{~s}$ ) for material 2 ( $130 \mathrm{~nm}$ ferrofluid) at various applied magnetic field gradient. B) Variation of normalized scattering intensity (normalized at $\mathrm{t}=30 \mathrm{~s}$ ) for material I ( $250 \mathrm{~nm}$ ferrofluid) at various applied magnetic field gradient. 


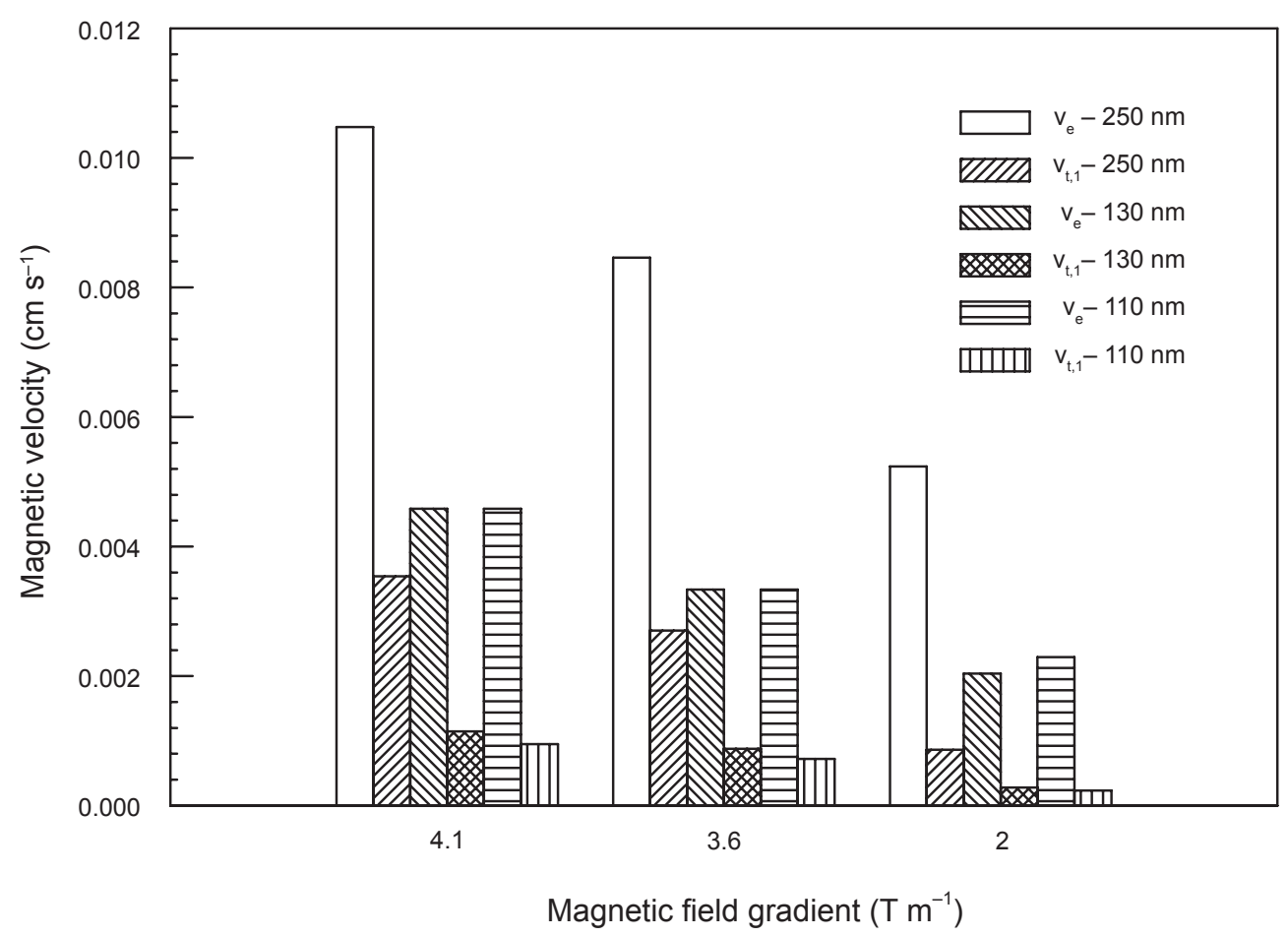

Figure 5 Comparison of experimentally measured velocity $\left(v_{e}\right)$ with theoretically predicted velocity based on single particle force balance $\left(v_{t, 1}\right)$ for different ferrofluid at various magnetic field gradients.

magnetic force. The experimentally measured velocity at a fixed magnetic field gradient is a function of mean primary particle size; hence, the velocity is higher for bigger particles. Further, for same particle sizes, the velocity is a function of the applied magnetic field gradient. These findings are consistent with the proposed model of force balance on chain-like agglomerates.

The experimentally estimated velocities were used to estimate the average number of primary particles in each chain-like agglomerate based on Equation 6. The effective force acting on the agglomerates was determined using Equation 3 (Table 2). The number of primary particles in the agglomerate $(n)$ was found to be a function of the magnetic field gradient, the primary particle size, and its underlying material composition. The number of primary particles in an agglomerate decrease as the magnetic field gradient and mean primary particle size increases. The uncoated nanoparticle (material 3) was found to form larger agglomerates compared to the similar sized PEG-coated material (material 2). High resolution scanning electron micrographs were taken of all materials and provided direct evidence of chaining. Figure 6 shows the difference in particle alignment and distribution after drying inside and outside the high magnetic field gradient region. The particles that dried outside the magnetic field gradient appear in a more random orientation, while those dried in the field appear to chain and an agglomerate. The effective magnetic force is higher than the force calculated without any agglomeration by a factor $n$. As the number of primary particles in agglomerate is found to have an inverse relation with the applied magnetic field gradient, it can be concluded that even a small magnetic field gradient can generate a very high effective force on magnetic nanoparticles due to enhanced agglomeration.

\section{In vitro transport characteristic of ferrofluid in a gel model of the brain using light microscopy}

After taking images of the test tubes at $0,3,6,16,32$, and 48 hours, the data was analyzed and normalized with ImageJ. The normalized pixel values for each series of gels at a given time point was averaged and the results displayed in Figure 7. The inherent assumption in this analysis is that a higher concentration of particles in an image, whether they are in or out of the plane of focus, will contribute to a higher average pixel value. Therefore, the mean pixel value was used as a proxy for total particle concentration in a given image. As particles diffuse away from the original infusion site, the overall concentration of particles for any given area of the image will decrease, leading to a lower mean pixel value. The results in Figure 7 suggest 


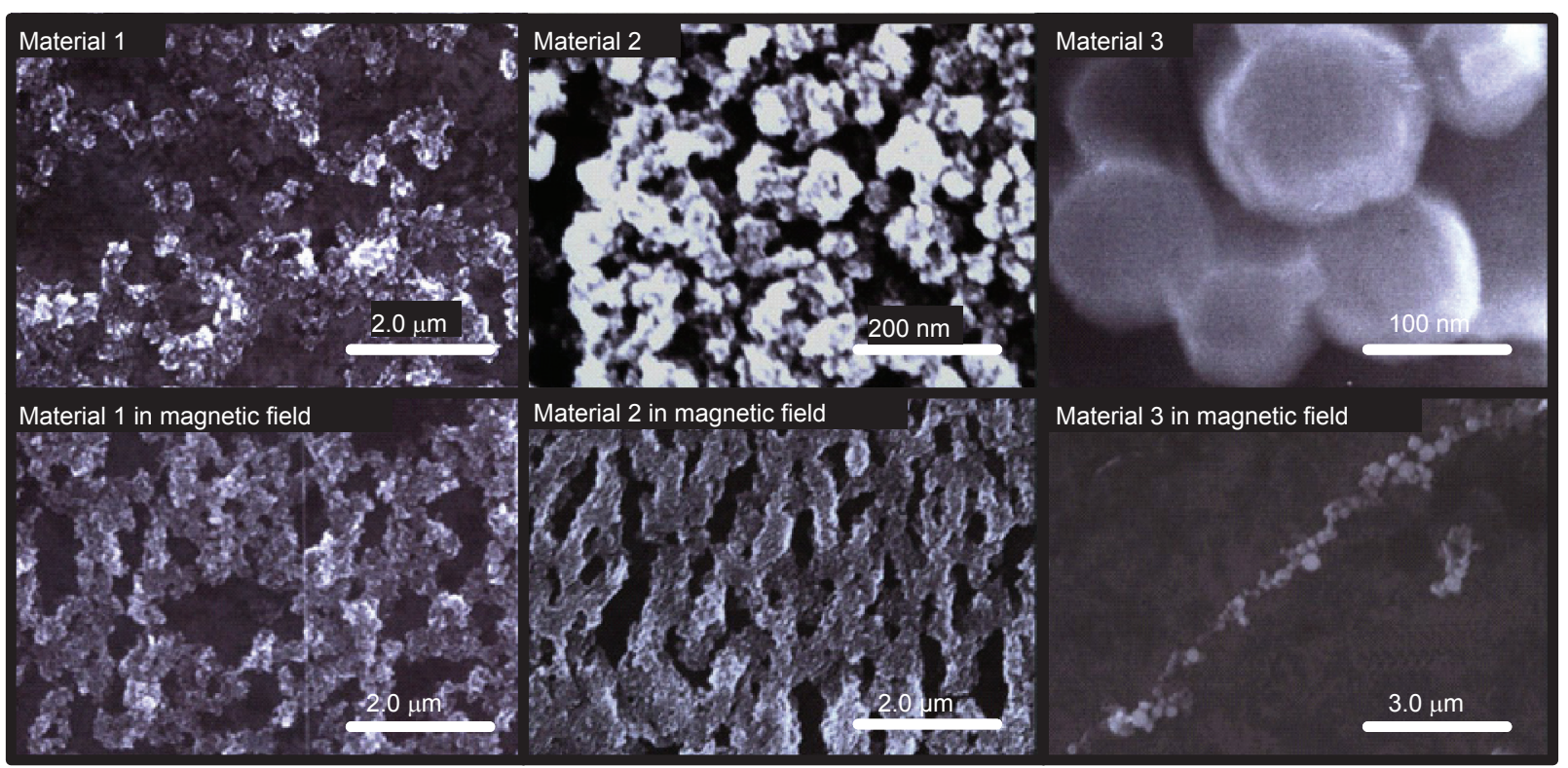

Figure 6 SEM images of different ferrofluids in the absence or presence of an external magnetic field.

that the highest magnetic field gradient $\left(8.0 \mathrm{~T} \mathrm{~m}^{-1}\right)$ retains more particles in the image. As the field strength decreases, the loss in average pixel value over time indicates diffusion of particles for both the materials. However, it is to be noted that, even a field as small as $0.55 \mathrm{~T} \mathrm{~m}^{-1}$ can be partially effective to hinder the free diffusion of the nanoparticle due to higher effective size as a result of collisional growth in the magnetic field gradient. One way to validate the above data is to examine the histograms of pixel values in the image over time (Figure S7). Initially stratified across the spectrum of pixel values, after 48 hours the control gel appears to converge, most likely onto the background (baseline) value. The gel exposed

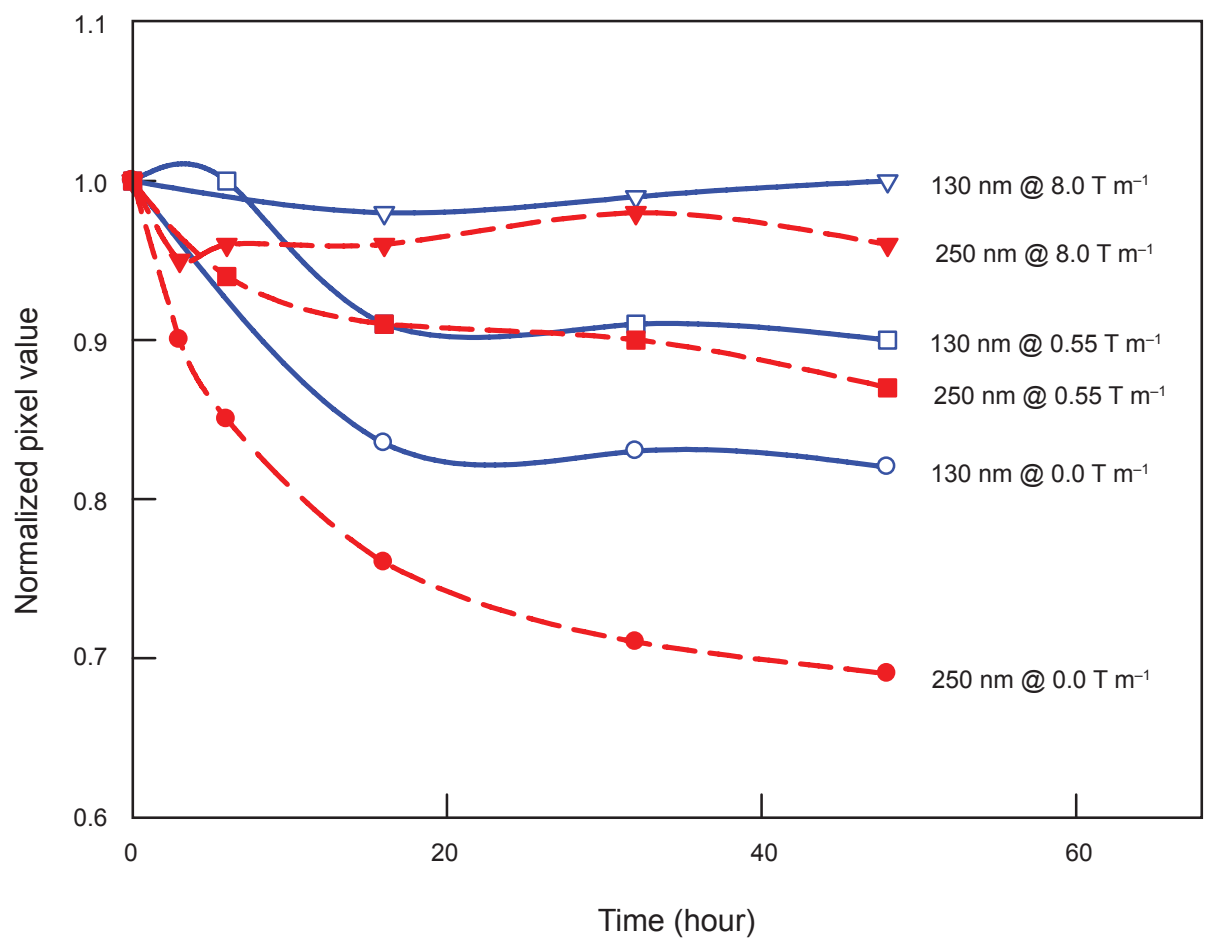

Figure 7 Plot of average normalized pixel values for gels with different size particles as a function of time and external magnetic field gradient. 
to the magnetic field gradient however retains a remarkably similar distribution to the 0 hour histogram. This would seem to indicate that the particles remain concentrated in the presence of the magnetic field gradient, in agreement with the data in Figure 7. Further, the decrease in normalized pixel value for $250 \mathrm{~nm}$ particles is greater compared to $130 \mathrm{~nm}$ particles at the same applied field, which may be an artifact of the experiments. Since the experiment is designed with the same initial concentration of ferrofluid $\left(1 \mathrm{mg} \mathrm{ml}^{-1}\right)$, the number concentration of the $130 \mathrm{~nm}$ sample is higher compared to the $250 \mathrm{~nm}$ samples. Due to higher overall projected surface area, the $130 \mathrm{~nm}$ sample contributes more towards the pixel value than the same amount of $250 \mathrm{~nm}$ sample.

Unfortunately, due to our desire to utilize the same particles analyzed in the experiments for velocity measurements, diffusion analysis was not done with one of the more accepted, and analytically rigorous methods proposed in the literature, such as fluorescence recovery after photobleaching (FRAP). ${ }^{28,29}$ Because of this, transport of particles in the gels is compared only qualitatively using an optical imaging technique. However, the proposed model clearly indicates that particles with a larger effective size will experience significant reductions in transport (diffusivity) in the magnetic field gradient due to agglomeration. The assertion that the particles are agglomerating is supported by the SEM results (Figure 6), as well as the high magnification light microscope results (Figure S8).

The results described seem to indicate that bulk flow of particles due to magnetic fields, as demonstrated by Kuhn and Holligan at $45.0 \mathrm{~T} \mathrm{~m}^{-1}$, does not occur in our in vitro model at gradients less than $10.0 \mathrm{~T} \mathrm{~m}^{-1}$. However, Goodwin ${ }^{4}$ noted that after a two hour magnetic exposure, the distribution of particles remained approximately the same for at least a further two hours after removing the magnet, indicating hindered diffusion. Goodwin's findings ${ }^{4}$ agree with the results of the diffusion experiments outlined above. The effective size of the particles is drastically increased by collisional growth forming a chain-like agglomerate as demonstrated in previous sections. Therefore, in our experiments the agglomerate of particles appears to diffuse much more slowly, hindering the diffusion of particles. The dependence of size on diffusivity has been confirmed with various in vivo and in vitro studies. ${ }^{11,30,31}$ Ohata and colleagues ${ }^{28}$ demonstrated that diffusion plays a role in spread of macromolecules through the cortex, and this phenomenon is dependent on size. In fact, Kuhn ${ }^{19}$ characterized the movement of superparamagnetic nanoparticles through extracellular matrix from murine sarcoma and found that the velocity of $135 \mathrm{~nm}$ particles was more than 100 times that of $400 \mathrm{~nm}$ particles.
This has tremendous implications for therapeutic use in the brain. In theory, small magnetic nanoparticles, approximately $50 \mathrm{~nm}$ or below, could be infused via CED to cover a large percentage of the intended target, as demonstrated by Muldoon. ${ }^{29}$ Then, the particles might be effectively locked into place by exposing the region to a high magnetic field gradient. The resulting agglomeration due to collisions in the magnetic field gradient increase the effective size and slow or prevent further passive diffusion of the particles. If diffusion plays a dominant role in the clearance of the particles, this could prove to be a method to significantly increase the local concentration. Even when diffusion is not the primary mode of clearance, agglomeration within the magnetic field gradient could play a role by inhibiting the convective process. Results from the velocity experiments suggest that the agarose fibers in a $0.6 \%$ agarose gel provide an effective barrier to movement of particles greater than or equal to $130 \mathrm{~nm}$ in diameter within a magnetic field gradient. $\mathrm{Chen}^{21}$ showed that the hydraulic conductivity was actually greater in the agarose model than in porcine brain, which suggests that the inability to move the nanoparticles might also occur with the in vivo situation.

However, given the diffusion and conductivity differences between different parts of the brain, the success of this inhibition could vary depending on the area in which it is deployed and the specific extracellular geometry of that region. Conversely, if smaller particles are infused via CED, it might be possible to maneuver them through the brain tissue using magnetic fields. Muldoon and colleagues ${ }^{29}$ found that 60-185 nm ferumoxide particles did not leak into rat tumors, while 20-50 nm particles did. The CED of $40 \mathrm{~nm}$ and $90 \mathrm{~nm}$ liposomes into glioma xenografts was detailed by Mamot and colleagues. ${ }^{11}$ The $40 \mathrm{~nm}$ liposomes were distributed throughout most of the tumor, and this effect was enhanced with the addition of mannitol, causing the liposomes to spread beyond the tumor boundaries. The $90 \mathrm{~nm}$ liposomes achieved just over $50 \%$ coverage after the addition of mannitol, suggesting that larger sizes reduced spread. The particle's size, coating, and magnetic properties will all be factors that could determine whether smaller particles aggregate and demonstrate decreased diffusion, or whether the particles might be actively moved with magnetic field manipulation. The gradient strength could also be an important factor in determining the tradeoff between these two possibilities.

\section{Conclusions}

A technique to determine the velocity of particles when a ferrofluid is exposed to magnetic fields was established. 
It has been demonstrated that the velocity of magnetic nanoparticles suspended in a fluid medium when exposed to a magnetic field gradient is enhanced due to an increase in effective size by collisional growth. A model was developed and used to estimate the effective size and the effective magnetic force acting on the agglomerate based on force balance on chain-like agglomerate structures. Further, it has been demonstrated that magnetic nanoparticles greater than or equal to $130 \mathrm{~nm}$ in diameter prove very difficult to guide through an in vitro model of the brain tissue. Result suggests that low magnetic field gradients (below $10 \mathrm{~T} \mathrm{~m}^{-1}$ ) could be used to limit passive diffusion, and possibly slow convection of nanoparticles within the brain due to agglomeration of particles. This could have many therapeutic benefits when combined with convection enhanced delivery of magnetic nanoparticles. Future work should be carried out with a more quantitative approach to measuring diffusion, and an in vivo model should be used to validate the results of the agarose gel.

\section{Acknowledgments}

This work was partially supported by a grant from the US Department of Defense (AFOSR) MURI Grant, FA9550-04-1-0430 and the Center of Materials Innovation, Washington University in St. Louis. David Brogan was supported by an Alpha Omega Alpha Carolyn L. Kuckein Student Research Fellowship.

\section{Nomenclature and Greek characters}

$B$, magnetic flux density $(T) ; d_{n}$, diameter of the sphere with same projected area as the area of the particle projected normal to its direction of motion $(m) ; d_{p}$, mean diameter of primary nanoparticle $(m) ; d_{s}$, diameter of the sphere whose effective surface equals that of particle $(m) ; F_{d, c}$, drag force acting on a chain-like agglomerate with n number of particles of diameter $d_{p}(N) ; F_{m, c}$, magnetic force acting on a chain-like agglomerate with $n$ number of particles of diameter $d_{p}(N)$; $k_{B}$, Boltzmann constant $\left(J K^{-1}\right) ; n$, average number of primary particles in each agglomerate; $N_{\mathrm{o}}$, initial number concentration of nanoparticles $\left(\# m^{-3}\right) ; T$, temperature $(K) ; v_{t, 1}$, terminal velocity in magnetic field gradient calculated based on single particle force balance $\left(\mathrm{m} \mathrm{s}^{-1}\right) ; v_{t, c}$, terminal velocity of a chainlike agglomerate with n number of particles of diameter $d_{p}$ in magnetic field gradient $\left(m \mathrm{~s}^{-1}\right) ; \beta_{F M}$, the collision frequency function in free molecular regime assuming the interacting particles form a chain-like agglomeration in a magnetic field gradient $\left(\# \mathrm{~s}^{-1}\right) ; \delta B$, magnetic field gradient $\left(T \mathrm{~m}^{-1}\right)$; $\eta$, viscosity of the fluid medium into which the particles are injected $\left(N s \mathrm{~m}^{-2}\right) ; \mu_{\mathrm{o}}$, permeability of free space; $\xi$, ratio of terminal velocity in magnetic field gradient calculated based on force balance on chain-like structure to the velocity calculated based on force balance on single particle $\left(v_{t, c} / v_{t, l}\right)$; $\rho_{p}$, density of the nanoparticles $\left(\mathrm{kg} \mathrm{m}^{-3}\right) ; \tau_{c}$, characteristic time for magnetic coagulation $\left(\mathrm{s}^{-1}\right) ; \tau_{t}$, characteristic time for terminal velocity $\left(s^{-1}\right) ; \chi_{v}$, volumetric magnetic susceptibility of the nanoparticle.

\section{References}

1. Takeda S, Mishima F, Fujimoto S, Izumi Y, Nishijima S. Development of magnetically targeted drug delivery system using superconducting magnet. J Magn Magn Mater. 2007;311:367-371.

2. Chen HT, Kaminski MD, Pytel P, MacDonald L, Rosengart AJ. Capture of magnetic carriers within large arteries using external magnetic fields. J Drug Target. 2008;16:262-268.

3. Alexiou C, Arnold W, Klein RJ, et al. Locoregional cancer treatment with magnetic drug targeting. Cancer Res. 2000;60:6641-6648.

4. Goodwin S, Peterson C, Hoh C, Bittner C. Targeting and retention of magnetic targeted carriers (MTCs) enhancing intra-arterial chemotherapy. J Magn Magn Mater. 1999;194:132-139.

5. Lubbe AS, Alexiou C, Bergemann C. Clinical experiences with magnetic drug targeting: A phase I study with 4'-epidoxorubicin in 14 patients with advanced solid tumors. Cancer Res. 1996;56:4686-4693.

6. Haffeli UO. Magnetically modulated therapeutic systems. Int J Pharm. 2004;277:19-24.

7. Lubbe AS, Alexiou C, Bergemann C. Clinical applications of magnetic drug targeting. J Surg Res. 2001;95:200-206.

8. Mykhaylyk O, Dudchenko N, Dudchenko A. Doxorubicin magnetic conjugate targeting upon intravenous injection into mice: High gradient magnetic field inhibits the clearance of nanoparticles from the blood. J Magn Magn Mater. 2005;293:473-482.

9. Fiandaca M, Forsayeth J, Banklewiez K. Current status of gene therapy trials for Parkinson's disease. Exper Neurol. 2008;209(1):51-57.

10. Chen ZJ, Broaddus WC, Viswanathan RR, Raghavan R, Gillies GT. Intraparenchymal drug delivery via positive-pressure infusion: Experimental and modeling studies of poroelasticity in brain phantom gels. IEEE Trans Biomed Eng. 2002;49:85-96.

11. Mamot C, Nguyen J, Pourdehnad M, et al. Extensive distribution of liposomes in rodent brains and brain tumors following convectionenhanced delivery. J Neurooncol. 2004;68:1-9.

12. Forbes ZG, Yellen BB, Barbee KA, Friedman G. Approach to targeted drug delivery based on uniform magnetic fields. IEEE Trans Magn. 2003;39:3372-3377.

13. Zborowski M, Fuh CB, Green R, Sun LP, Chalmers JJ. Analytical magnetapheresis of ferritin-labeled lymphocytes. Anal Chem. 1995;67:3702-3712.

14. Rousan AA, Elghanem HM, Yusuf NA. Faraday-rotation and chain formation in magnetic fluids. IEEE Trans Magn. 1989;25:3121-3124.

15. Nair SS, Rajesh S, Abraham VS, Anantharaman MR, Nampoori VPN. Magnetic field-induced cluster formation and variation of magnetooptical signals in zinc-substituted ferrofluids. J Magn Magn Mater. 2006;305:28-34.

16. Relle S, Grant SB, Tsouris C. Diffusional coagulation of superparamagnetic particles in the presence of an external magnetic field. Physica A. 1999;270:427-443.

17. Corr SA, Byrne SJ, Tekoriute R, et al. Linear assemblies of magnetic nanoparticles as MRI contrast agents. J Am Chem Soc. 2008;130:4214-4215.

18. Holligan DL, Gillies GT, Dailey JP. Magnetic guidance of ferrofluidic nanoparticles in an in vitro model of intraocular retinal repair. Nanotechnology. 2003;14:661-666. 
19. Kuhn SJ, Hallahan DE, Giorgio TD. Characterization of superparamagnetic nanoparticle interactions with extracellular matrix in an in vitro system. Ann Biomed Eng. 2006;34:51-58.

20. Kulkarni P, Dutari G, Biswas P, Haught R. Gravity settling characteristics of Cryptosporidium parvum oocysts in aqueous suspension using in situ static light scattering. Colloids Surf A Physicochem Eng Asp 2004;233: (1-3):1-10.

21. Chen Z, Gillies G, Broaddus W, et al. A realistic brain tissue phantom for intraparenchymal infusion studies. J Neurosurg. 2004;101:314-322.

22. Gries G, Guiseley K, Serwer P. The relationship of agarose gel structure to the sieving of spheres during agarose gel electrophoresis. Biophys $J$. 1993;65:138-148.

23. Fuchs NA. The mechanism of aerosols. Oxford, UK: Pergamon Press; 1964.

24. Gonda I, Abd El Khalik AF. On the calculation of aerodynamic diameters of fibers. Aerosol Sci Technol. 1985;4:233-238.
25. Leith D. Drag on nonspherical objects. Aerosol Sci Technol. 1987;6:153-161.

26. Kumar P, Biswas P. Analytical expressions of the collision frequency function for aggregation of magnetic particles. J Aerosol Sci. 2005;36:455-469.

27. Fernandes PRG, Mukai H, Laczkowski IM. Magneto-optical effect in lyotropic liquid crystal doped with ferrofluid. J Magn Magn Mater. 2005;289:115-117.

28. Ohata K, Marmarou A. Clearance of brain edema and macromolecules through the cortical extracellular space. J Neurosurg. 1992;77:387-396.

29. Muldoon L, Sandor M, Pinkston K, et al. Imaging, distribution, and toxicity of superparamagnetic iron oxide magnetic resonance nanoparticles in the rat brain and intracerebral tumor. Neurosurgery. 2005;57:785-796. 


\section{Supporting information Experimental setup for nanoparticle synthesis}

The experimental setup for synthesis of nanocomposite consisted of three components. A nebulizer was used as a precursor feeding system, a tubular furnace aerosol reactor (FuAR, Lindbergh BlueM model STF54779C, effective length $106 \mathrm{~cm}$ and internal diameter $2.5 \mathrm{~cm}$ ) was used to decompose the precursor and an electrostatic precipitator (ESP) was used for particle collection. Aqueous solution of $\mathrm{Fe}\left(\mathrm{NO}_{3}\right)_{3}$ and $\mathrm{CuSO}_{4}(2: 1$ molar ratio) was nebulized and carried by air into the FuAR and was decomposed at different temperatures $\left(1000-1200^{\circ} \mathrm{C}\right)$. Air at different flow rate was used to provide an oxidizing atmosphere, and to control the residence time inside the reactor. Precursor concentration $(0.1-1 \mathrm{M})$ was varied to produce particles between 70-200 nm mobility diameter. The synthesized particles were collected on a cylindrical ESP with applied potential of $+10 \mathrm{kV}$.

Particle mobility size distribution was measured at the exit of FuAR using a scanning mobility particle sizer (SMPS, Model: TSI 3936) system. The size and microstructures were verified by a SEM (Model JEOL 7001LVF FE-SEM). The crystallographic characteristics were analyzed by powder XRD technique (Model: Rigaku D-MAX/A9 diffractometer, $\mathrm{Cu} \mathrm{K} \alpha$ radiation, $\lambda=1.5418 \AA$ ).

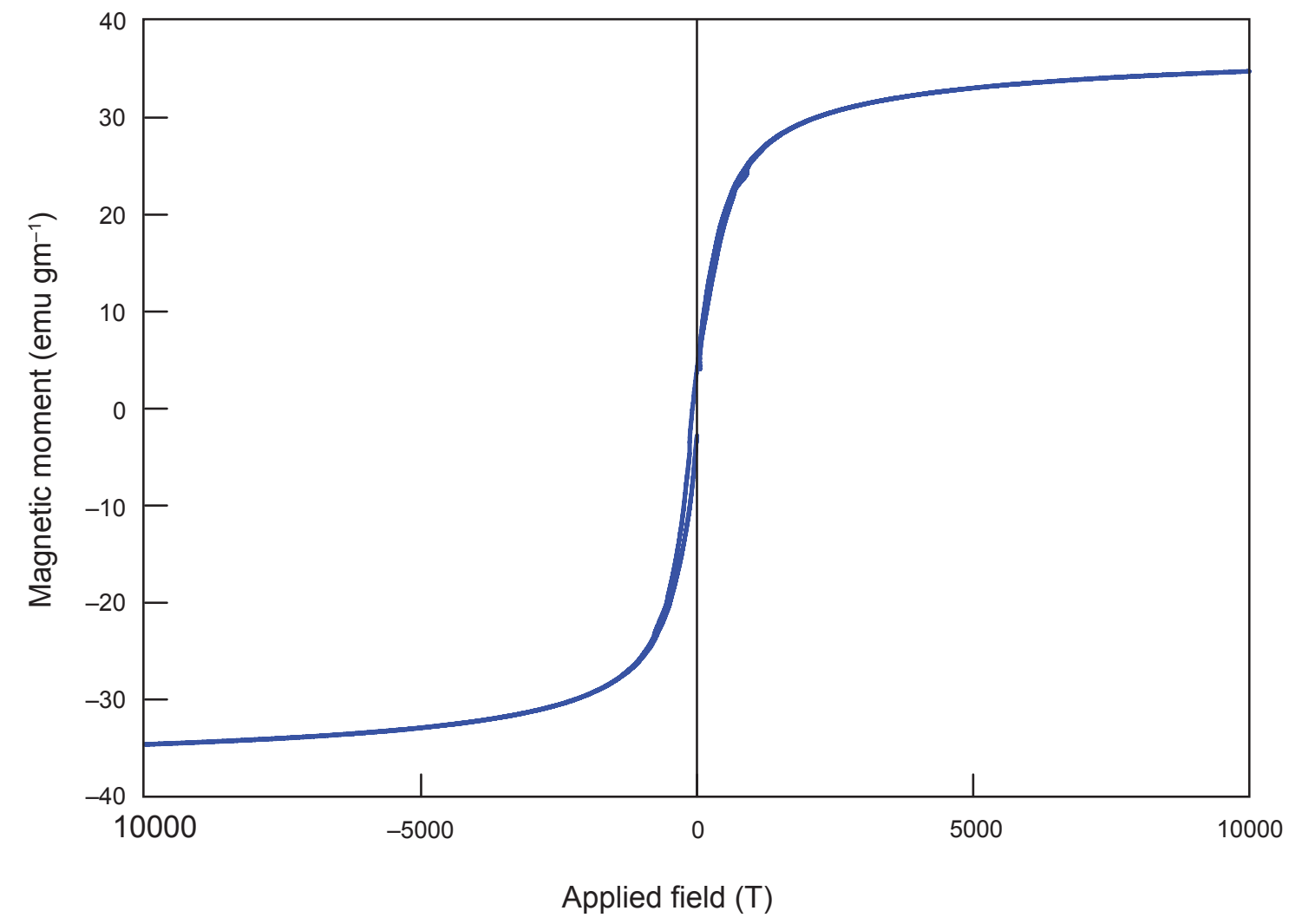

Figure SI Hysteresis loop for synthesized II $10 \mathrm{~nm} \mathrm{CuFe}{ }_{2} \mathrm{O}_{4}$ at $300 \mathrm{~K}$ measured using a Quantum Design PPMS SQUID (model 6000). 


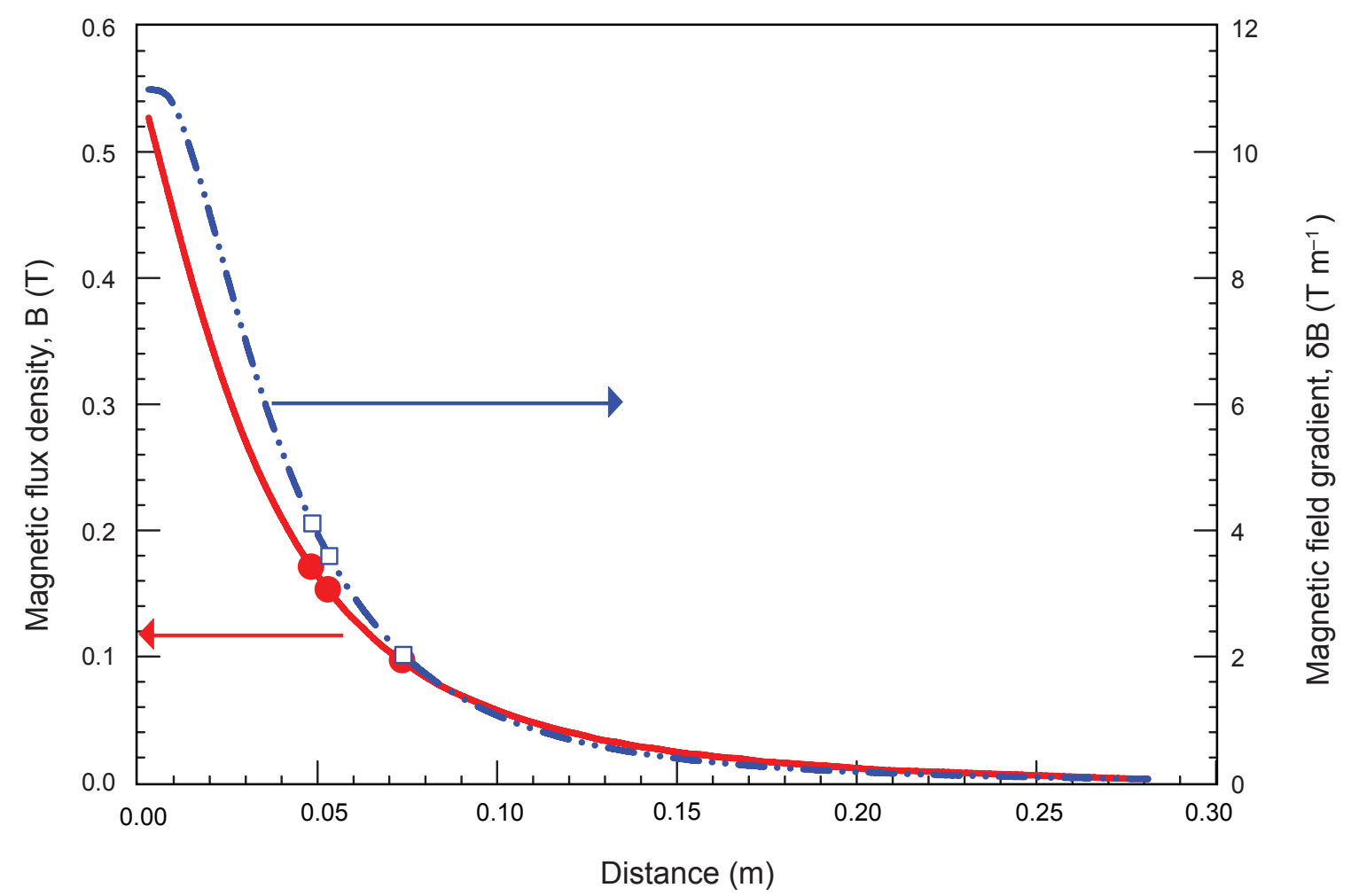

Figure S2 Magnetic flux density $(B)$ and magnetic field gradient $(\delta B)$ as a function of distance from the surface of the poles. The lines are representing the measurements with the gaussmeter, while the symbols represent the magnetic field gradients which were actually used in this study.

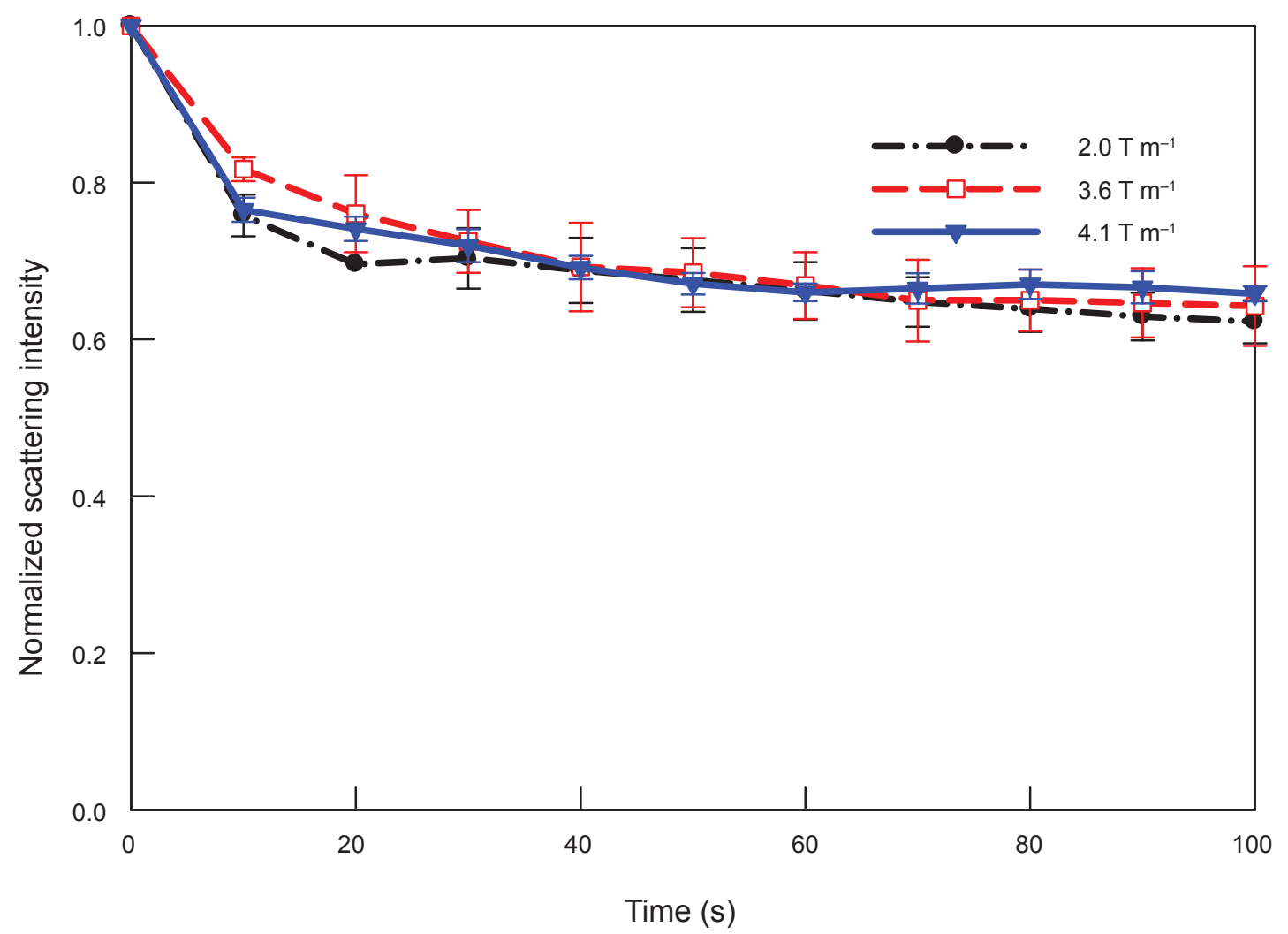

Figure S3 Variation of normalized scattering intensity (normalized at $\mathrm{t}=0 \mathrm{~s}$ ) due to magnetic coagulation for material 3 (I I0 nm ferrofluid) at various applied magnetic field gradient. 


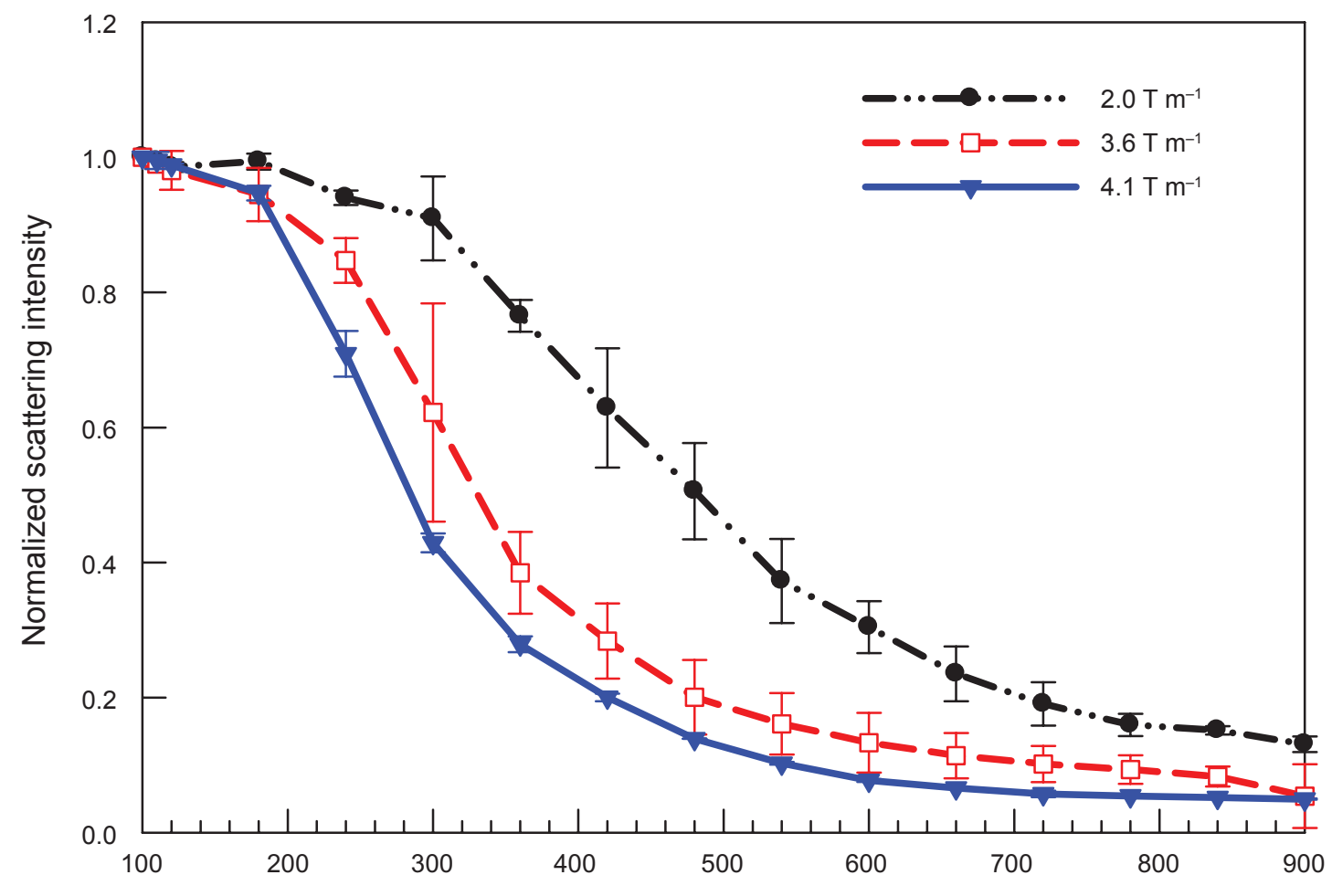

Time (s)

Figure S4 Variation of normalized scattering intensity (normalized at $\mathrm{t}=100 \mathrm{~s}$ ) due to velocity for material $3(110 \mathrm{~nm}$ ferrofluid) at various applied magnetic field gradient.

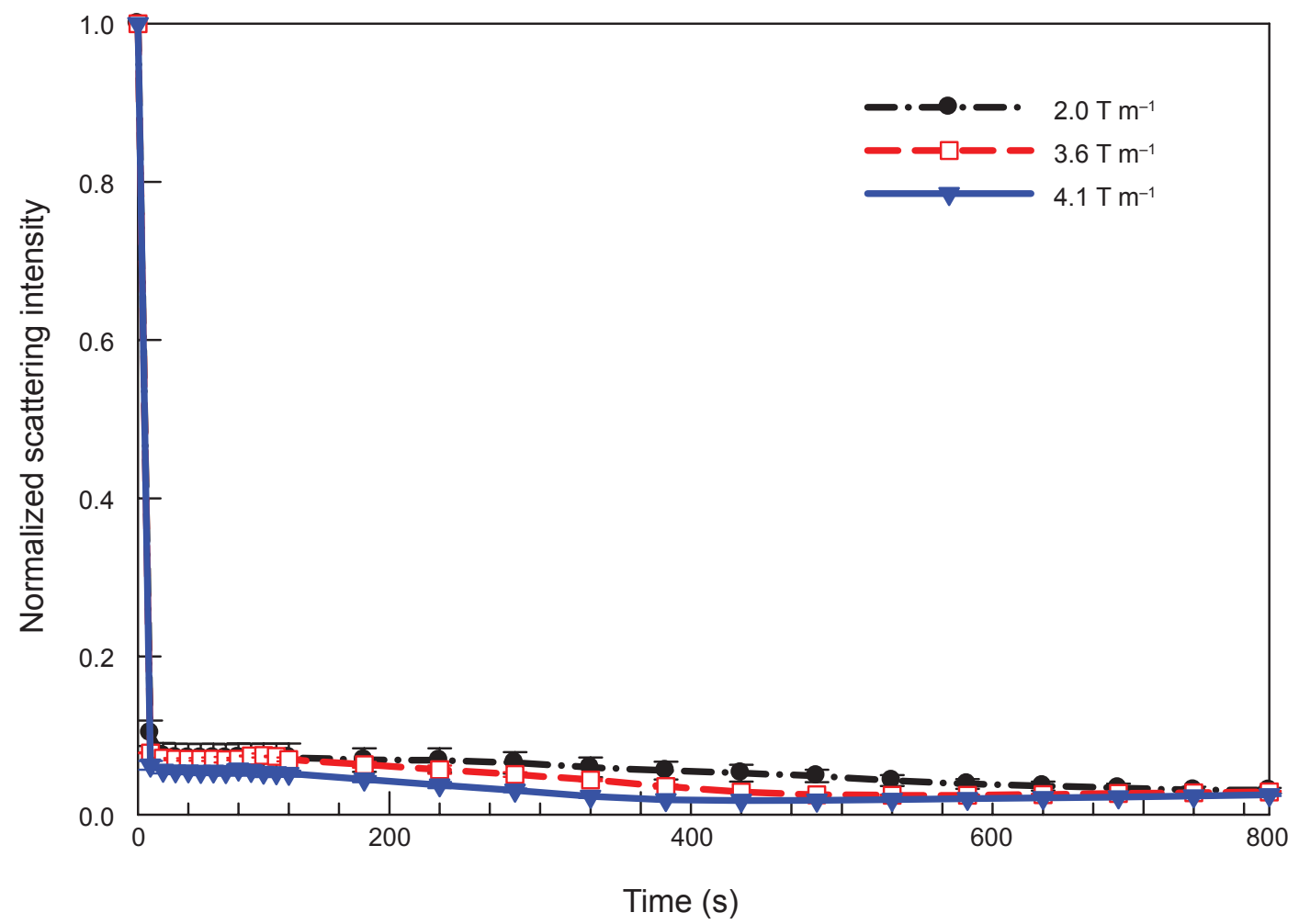

Figure S5 Variation of normalized scattering intensity (normalized at $\mathrm{t}=0 \mathrm{~s}$ ) with time for material $2(130 \mathrm{~nm}$ ferrofluid) at various applied magnetic field gradient. 


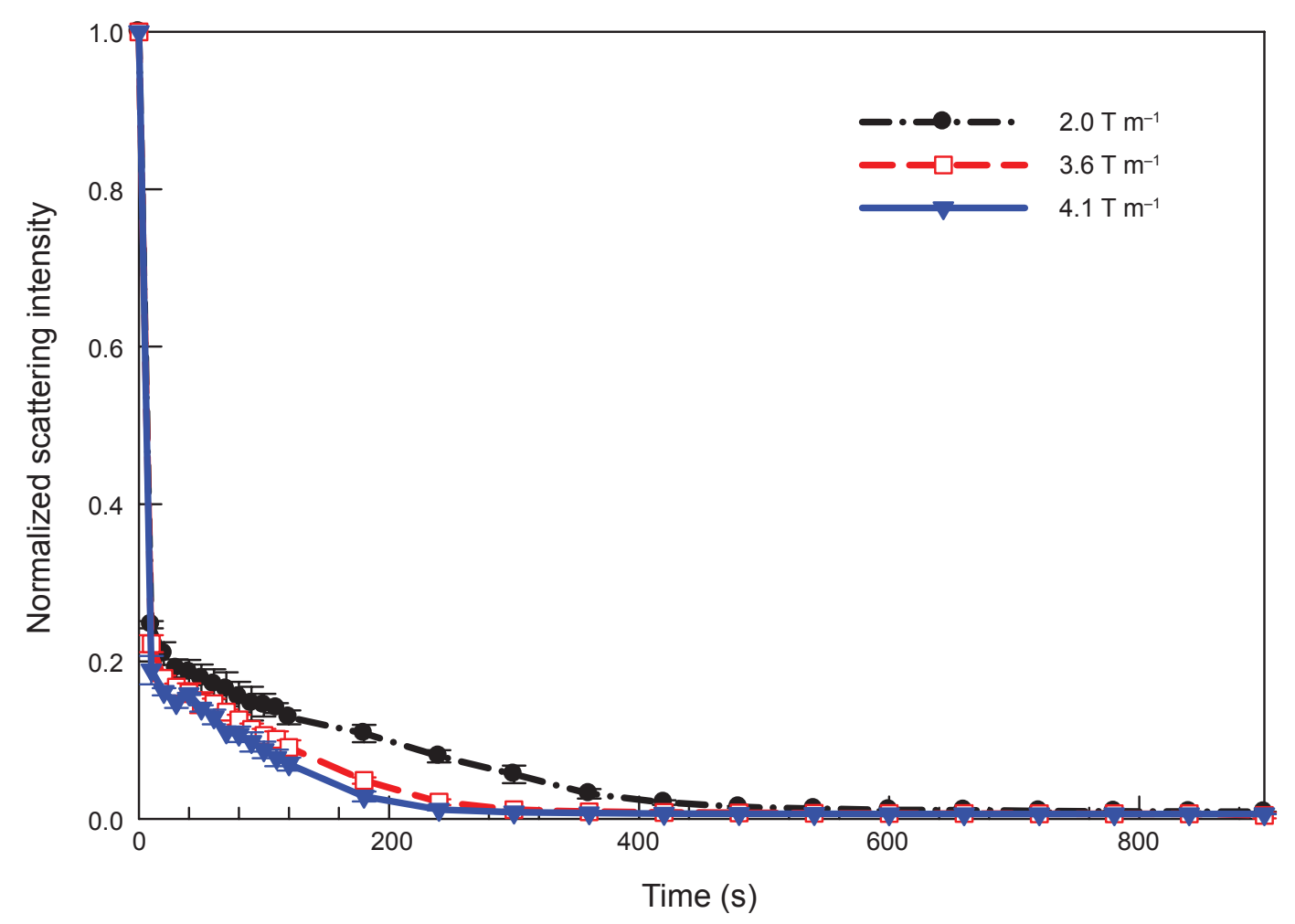

Figure S6 Variation of normalized scattering intensity (normalized at $t=0 \mathrm{~s}$ ) with time for material I $(250$ nm ferrofluid) at various applied magnetic field gradient.
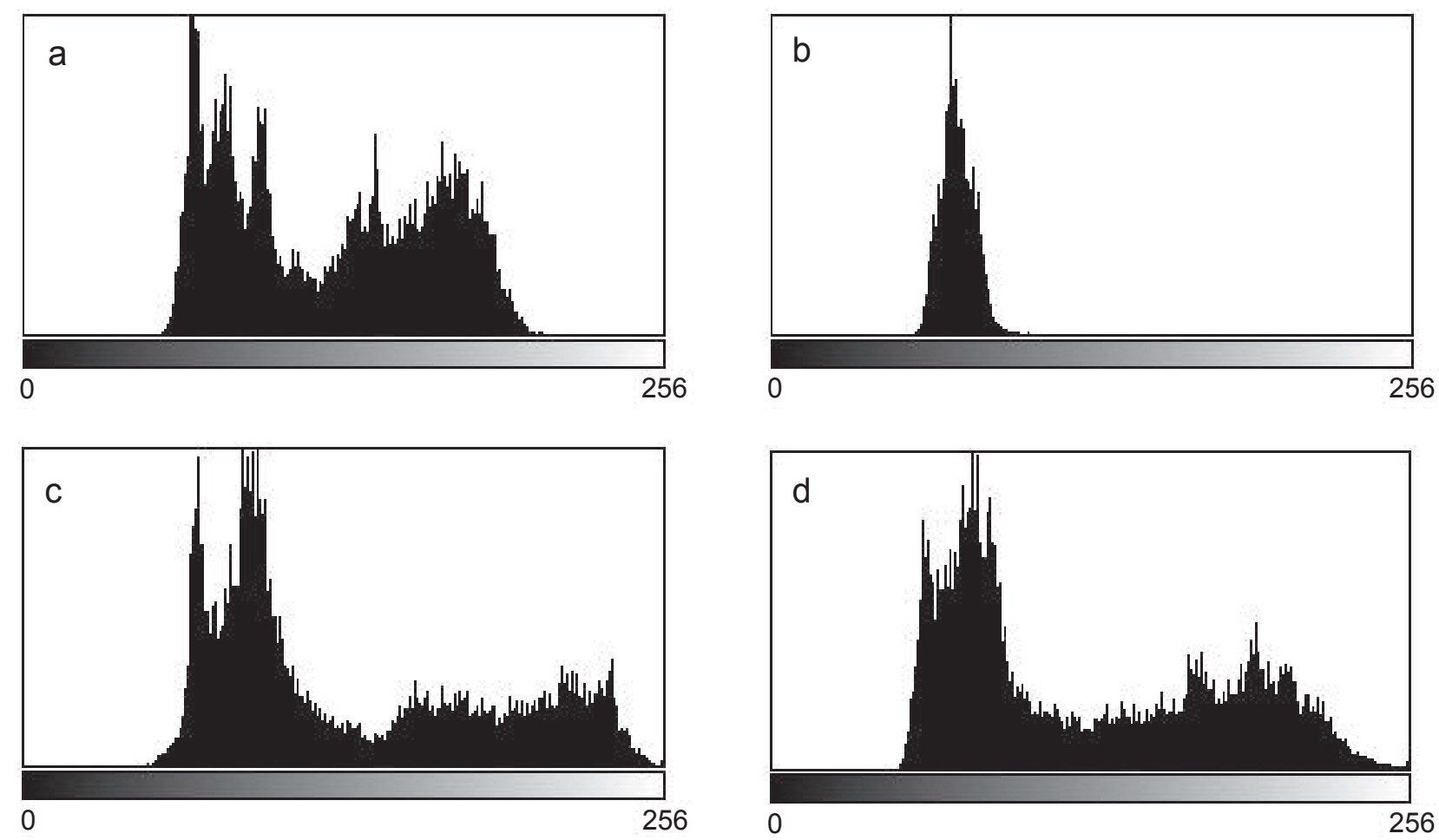

Figure S7 Histograms of control gel infused with $130 \mathrm{~nm}$ particles at 0 hrs (a) and 48 hrs (b), and gel infused with $130 \mathrm{~nm}$ particles at (c) 0 hrs and (d) after 48 hrs in the high magnetic field gradient $\left(8.0 \mathrm{Tm}^{-1}\right)$. 


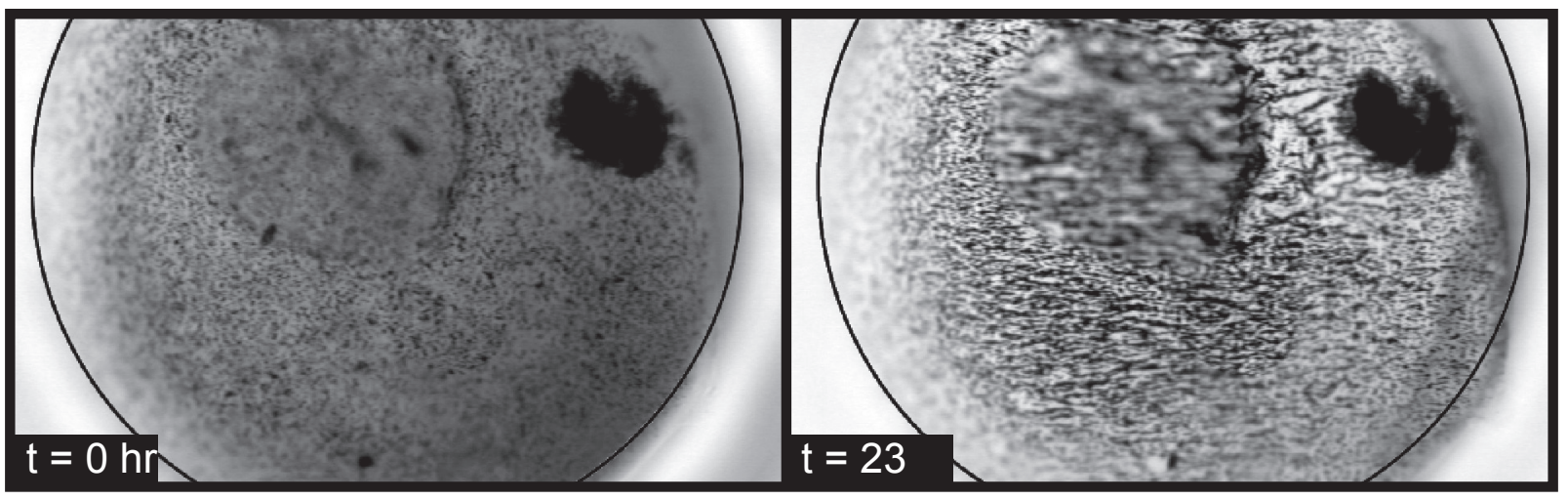

Figure $\mathbf{S 8}$ Images of $110 \mathrm{~nm}$ particles (material 3) placed into middle of gel after punching with a needle before being placed into the magnetic field (left) and after 23 hours in the magnetic field (right) 